\title{
EIGENSPACE ARRANGEMENTS OF REFLECTION GROUPS
}

\author{
ALEXANDER R. MILLER
}

\begin{abstract}
The lattice of intersections of reflecting hyperplanes of a complex reflection group $W$ may be considered as the poset of 1-eigenspaces of the elements of $W$. In this paper we replace 1 with an arbitrary eigenvalue and study the topology and homology representation of the resulting poset. After posing the main question of whether this poset is shellable, we show that all its upper intervals are geometric lattices and then answer the question in the affirmative for the infinite family $G(m, p, n)$ of complex reflection groups, and the first 31 of the 34 exceptional groups, by constructing CL-shellings. In addition, we completely determine when these eigenspaces of $W$ form a $K(\pi, 1)$ (resp. free) arrangement.

For the symmetric group, we also extend the combinatorial model available for its intersection lattice to all other eigenvalues by introducing balanced partition posets, presented as particular upper order ideals of Dowling lattices, study the representation afforded by the top (co)homology group, and give a simple map to the posets of pointed $d$-divisible partitions.
\end{abstract}

\section{Contents}

1. Main question and results

2. Preliminaries

3. General reductions

4. $G(m, p, n)$ case of Theorem 1.2

5. Maximal eigenspaces of $G(m, p, n)$

6. $G(m, p, n)$ case of Theorem 1.1

7. Exceptional cases of Theorems 1.1 and 1.2

8. Consequences for the topology of eigenspace arrangements

9. Combinatorics of $E(W, \zeta)$ in type $A_{n-1}$

10. Proof of Theorem 1.3

Acknowledgements

\section{MAIN QUESTION AND RESUlts}

Let $V$ denote an $n$-dimensional $\mathbb{C}$-vector space. A reflection in $V$ is any nonidentity element $r$ in $\operatorname{GL}(V)$ of finite order that fixes some hyperplane $H_{r}$, and a finite subgroup $W$ of $\mathrm{GL}(V)$ is called a reflection group if it is generated by reflections.

Received by the editors March 16, 2013 and, in revised form, October 14, 2013 and October 15, 2013.

2010 Mathematics Subject Classification. Primary 20F55, 05E10, 05E18, 55P20, 14 N20.

Key words and phrases. d-divisible partitions, Dowling lattices, Eilenberg-MacLane spaces, reflection groups, ribbon representations, subspace arrangements. 
Example. The action of the symmetric group $\mathfrak{S}_{n}$ on $[n]:=\{1,2, \ldots, n\}$ gives rise to a faithful action on $\mathbb{C}^{n}$ via $\sigma\left(e_{i}\right)=e_{\sigma(i)}$, where $e_{1}, e_{2}, \ldots, e_{n}$ denote the standard basis vectors. Since the transpositions act as reflections and generate the group, this representation realizes $\mathfrak{S}_{n}$ as a reflection group in $\mathrm{GL}\left(\mathbb{C}^{n}\right)$. We shall refer to it as the defining representation.

For an element $g \in W$ and root of unity $\zeta$, let $V(g, \zeta)$ denote the $\zeta$-eigenspace of $g$ in $V$. Define $E(W, \zeta)$ to be the $W$-poset (partially ordered set) of all such $\zeta$-eigenspaces $\{V(g, \zeta)\}_{g \in W}$ ordered by reverse inclusion, with $W$-action given by $h \cdot V(g, \zeta)=V\left(h g h^{-1}, \zeta\right)$. The eigenvalue $\zeta=1$ gives the lattice $\mathscr{L}_{W}$ of intersections of reflecting hyperplanes for $W$; see [29, Lemma 4.4]. The minimal elements of $E(W, \zeta)$ (i.e., the inclusion-maximal $\zeta$-eigenspaces for $W$ ) are the focus of Springer's theory of regular elements [35, and each has dimension equal to the number $a(d)$ of degrees $d_{1}, d_{2}, \ldots, d_{n}$ of $W$ that are divisible by $d$, the order of $\zeta$; see Proposition 3.7 below. When $W$ is crystallographic, the poset $E(W, \zeta)$ itself appears in Broué, Malle, and Michel's $\Phi$-Sylow theory [8].

Example. The degrees of $\mathfrak{S}_{4}$ are $1,2,3,4$, and those of the dihedral group $I_{2}(4)$ (whose cardinality is 8 ) are 2,4 . Hence the following table.

\begin{tabular}{lccccc}
\hline$d$ & 1 & 2 & 3 & 4 & $\geq 5$ \\
\hline$a(d)$ for $\mathfrak{S}_{4}$ & 4 & 2 & 1 & 1 & 0 \\
$a(d)$ for $I_{2}(4)$ & 2 & 2 & 0 & 1 & 0 \\
\hline
\end{tabular}

When $a(d)=0$, the poset $E(W, \zeta)$ has only one element, the 0-dimensional subspace. For all other listed cases, we have drawn the Hasse diagram of $E(W, \zeta)$ in Figures 15 and labeled each eigenspace by linear equations that define it. Maximal eigenspaces are numbered for easy reference. For example, the maximal eigenspace $E_{4}$ labeled (4) in Figure 3 is the $\zeta$-eigenspace for the 3 -cycle permutation $g=(1,4,3)$. Note that the $d=1,2$ cases for $I_{2}(4)$ coincide, since the scalar matrix -1 is an element of $I_{2}(4)$; see Corollary 3.8 below.

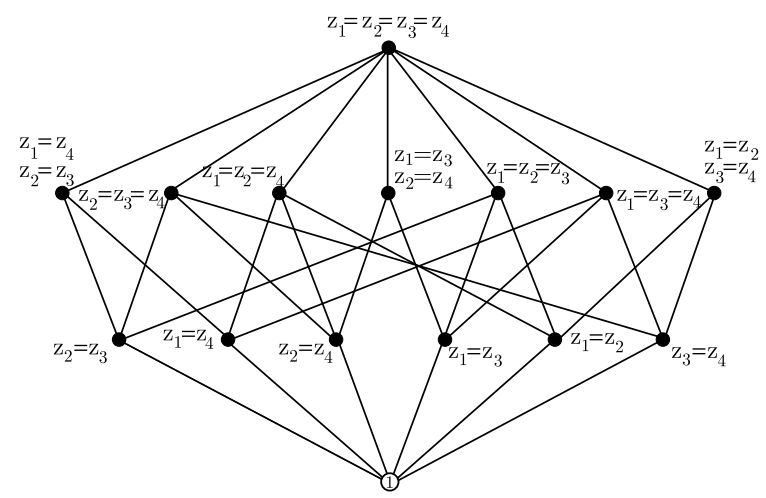

Figure 1. The poset $E\left(\mathfrak{S}_{4}, 1\right)=\mathscr{L}_{\mathfrak{S}_{4}}$ of 1 -eigenspaces for $\mathfrak{S}_{4} \subset \mathrm{GL}\left(\mathbb{C}^{4}\right)$. 


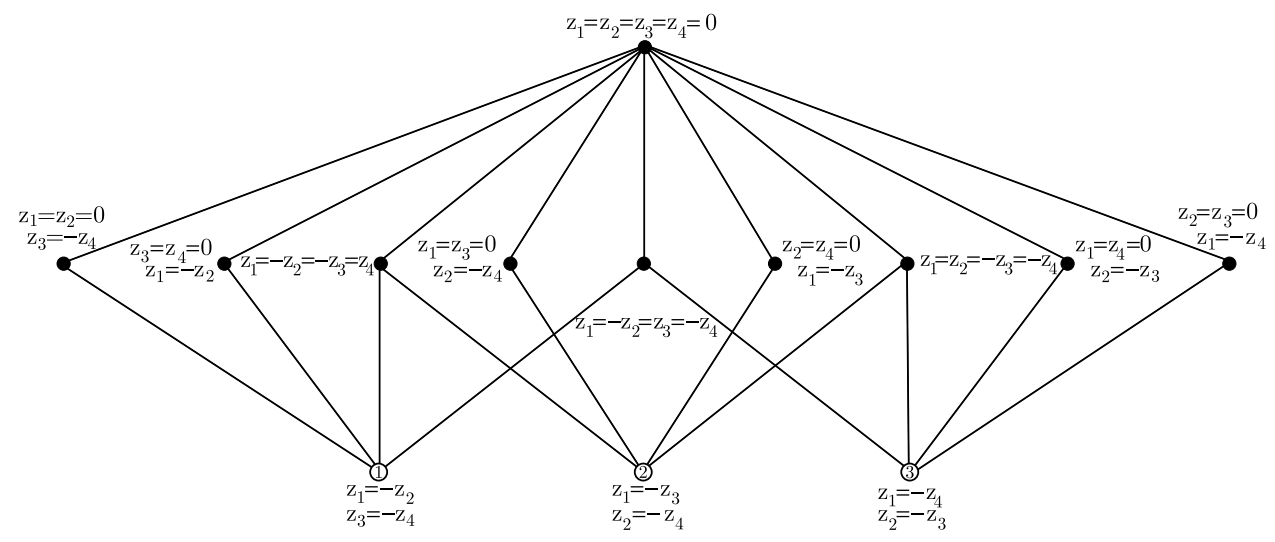

Figure 2. The poset $E\left(\mathfrak{S}_{4},-1\right)$ of $(-1)$-eigenspaces for $\mathfrak{S}_{4} \subset \mathrm{GL}\left(\mathbb{C}^{4}\right)$.

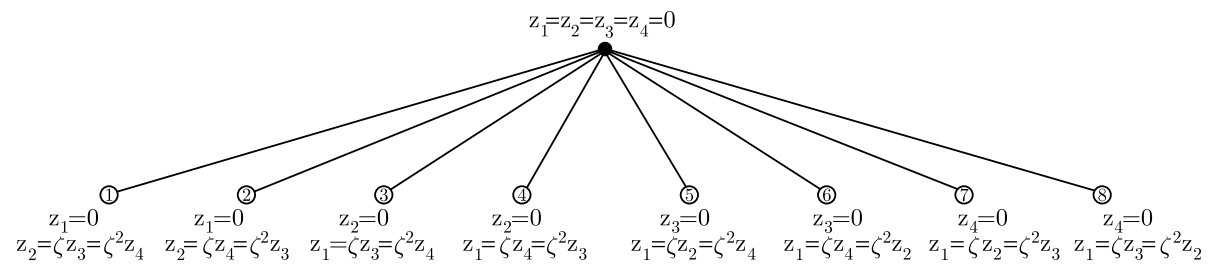

Figure 3. The poset $E\left(\mathfrak{S}_{4}, \zeta_{3}\right)$ of $\zeta$-eigenspaces for $\zeta$ any primitive 3 rd root of unity and $\mathfrak{S}_{4} \subset \mathrm{GL}\left(\mathbb{C}^{4}\right)$.

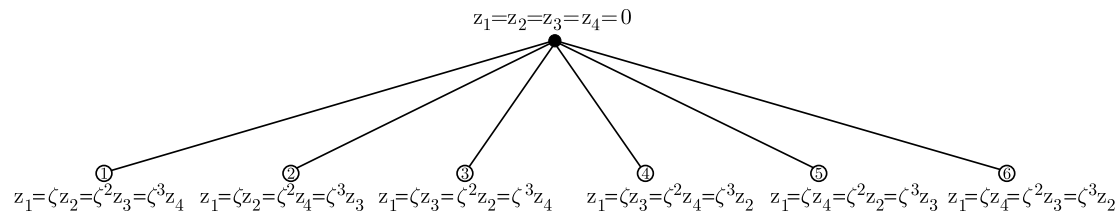

FIgure 4 . The poset $E\left(\mathfrak{S}_{4}, \zeta\right)$ of $\zeta$-eigenspaces for $\zeta$ any primitive th root of unity and $\mathfrak{S}_{4} \subset \mathrm{GL}\left(\mathbb{C}^{4}\right)$.
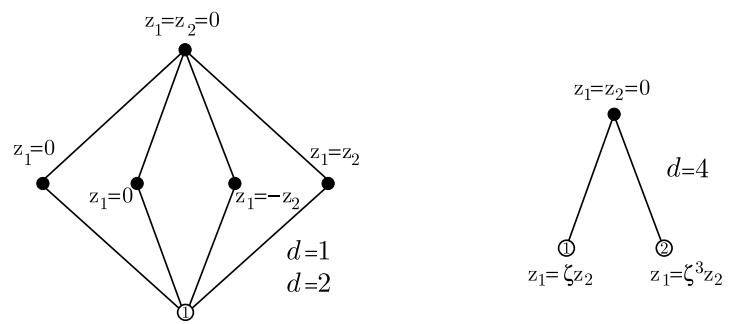

Figure 5. The poset $E(W, \zeta)$ of $\zeta$-eigenspaces for the dihedral group $I_{2}(4)$ of order 8 , and $\zeta$ of order $d=1,2,4$. 
This paper concerns the following problem of Lehrer and Taylor [25, Problem 7].

Problem (Lehrer-Taylor). Study connections between the structure and representations of $W$ and the topology of the posets $E(W, \zeta)$.

In the case of $E\left(\mathfrak{S}_{n}, 1\right)$, Stanley [38 used the work of Hanlon [17] to obtain an explicit expression for the top homology representation, which combined with Klyachko's work to establish a connection with the Lie representation $\mathrm{Lie}_{n}$; see 19 , 21]. Lehrer and Solomon [22] extended Stanley's result and conjectured an analogue for all other finite Coxeter groups. Hanlon's work [18] on Dowling lattices gives an alternate extension and provides the top homology character of $E(W, 1)$ for $W=G(m, 1, n)$, the complex reflection group of $n \times n$ monomial matrices whose nonzero entries are $m^{\text {th }}$ roots of unity. N. Bergeron 1] gave a type $B$ analogue of the above-mentioned Lie correspondence that was later generalized to Dowling lattices by Gottlieb and Wachs [16]. In addition to the above, analogous results have been obtained for various subposets of $E\left(\mathfrak{S}_{n}, 1\right)$; see [45]. However, the author is unaware of any analogous results for $\zeta \neq 1$, even for $\mathfrak{S}_{n}$.

Question A. Is the following true for every $\zeta$ and every reflection group $W$ ?

(Weak version) $E(W, \zeta)$ is homotopy Cohen-Macaulay.

(Strong version) $\widehat{E(W, \zeta)}$ is CL-shellable.

The fact that CL-shellability implies homotopy CM-ness is well known; see $\$ 2$,

Our first main result answers affirmatively the strong version of Question A for all irreducible complex reflection groups except types $E_{6}, E_{7}, E_{8}$ (which, in the Shephard-Todd classification, are $G_{35}, G_{36}, G_{37}$ ). Since the question reduces (see $\$ 3$ below) to the case where $W$ acts irreducibly, only these three Weyl groups remain.

Theorem 1.1. Let $W=G(m, p, n)$ or one of the first 31 exceptional groups $G_{4}, G_{5}, \ldots, G_{34}$, and let $\zeta$ be a primitive $d^{\text {th }}$ root of unity. Then $\widehat{E(W, \zeta)}$ is $C L$ shellable. In particular, the order complex $\Delta(\overline{E(W, \zeta)})$ is a pure bouquet of spheres.

Our second main result is central to the first.

Theorem 1.2. Let $W$ be a reflection group and let $\zeta$ be a primitive $d^{\text {th }}$ root of unity. Then

$$
E(W, \zeta)=\left\{E \cap X: X \in \mathscr{L}_{W} \text { and } E \in E(W, \zeta) \text { maximal }\right\} .
$$

In particular, each upper interval $[x, \hat{1}]$ in $E(W, \zeta)$ is a geometric lattice.

We will see in Section 3 below that Theorem 1.2 also has interesting consequences of its own, namely, that $E(W, \zeta)$ depends only on $d$ (see Theorem 7.1 for a much sharper result) and is built from copies (conjugates) of the intersection lattice $\mathscr{L}_{W(d)}$ of Lehrer and Springer's reflection subquotient $W(d)$ (see Proposition 3.9).

Remark. The arrangement of $\zeta$-eigenspaces is not always so well behaved for nonreflection groups $G \subset \mathrm{GL}\left(\mathbb{C}^{n}\right)$. For example, take $G$ to be $\mathbb{Z} / 3 \mathbb{Z} \times \mathfrak{S}_{4}$ acting faithfully on $\mathbb{C}^{4}$ by $(i, \sigma)\left(e_{j}\right)=\zeta^{i} e_{\sigma(j)}$ for some primitive cube root of unity $\zeta$. Then the set of all 1-eigenspaces for $G$ is just the union of the 1-, $\zeta$-, and $\zeta^{2}$-eigenspaces for the defining representation of $\mathfrak{S}_{4}$ that we wrote down in Figure 1 and Figure 3 above. The end result is the Hasse diagram of $E(G, 1)$ in Figure [6] which shows that for this non-reflection group $G \subset \mathrm{GL}\left(\mathbb{C}^{4}\right)$, the poset of 1-eigenspaces $E(G, 1)$ is not Cohen-Macaulay, and its upper intervals are not all geometric lattices. 


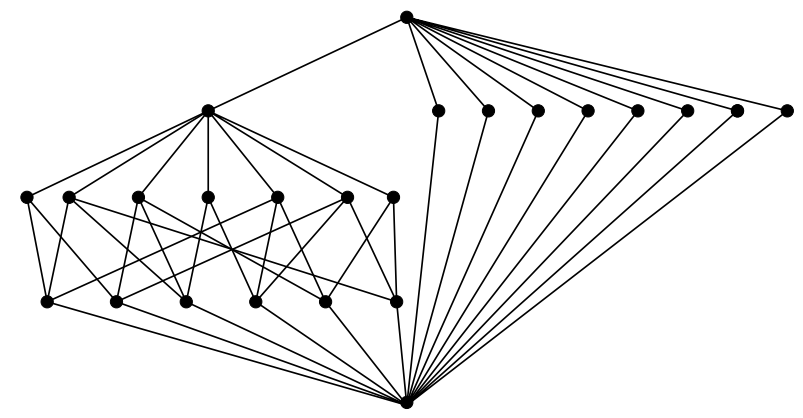

Figure 6 . The poset of 1-eigenspaces for the group $G$ in the preceding remark that falsifies the conclusion of both Theorem 1.1 and Theorem 1.2 for a non-reflection group.

We also characterize when an eigenspace arrangement of a reflection group comes from an arrangement of hyperplanes (Lemma 10.3 below). As a side benefit, this will answer the natural $K(\pi, 1)$ and freeness questions for eigenspaces. That is, we determine exactly when the complement of the proper $\zeta$-eigenspaces of a reflection group $W$ is $K(\pi, 1)$, and exactly when the $\zeta$-eigenspaces of codimension 1 form a free (hyperplane) arrangement. For brevity, define $\mathcal{A}(W, d)$ to be the set of all proper $\zeta$-eigenspaces of the reflection group $W$ that are maximal under inclusion for a fixed but arbitrary choice of primitive $d^{\text {th }}$ root of unity $\zeta$, so that the complement of the proper $\zeta$-eigenspaces of $W$ is

$$
\mathcal{M}(W, d)=\mathbb{C}^{n} \backslash \bigcup_{X \in \mathcal{A}(W, d)} X .
$$

The two questions were resolved affirmatively for the highly nontrivial case of the reflection arrangement $\mathcal{A}(W, 1)$ by Bessis and Terao, respectively, and in contrast to Question $\mathrm{A}$ the non-classical case $\mathcal{A}(W, d) \neq \mathcal{A}(W, 1)$ is surprisingly simple.

Theorem 1.3. Let $W \subset \mathrm{GL}\left(\mathbb{C}^{n}\right)$ be a complex reflection group and let $d>0$.

Then the following are equivalent:

(i) One has $a(d)=n$ or $a(d)=n-1$. That is, $\mathcal{A}(W, d)$ contains a hyperplane.

(ii) The arrangement $\mathcal{A}(W, d)$ is a free hyperplane arrangement.

(iii) The complement $\mathcal{M}(W, d)$ is a $K(\pi, 1)$ space.

Furthermore, $\mathcal{A}(W, d)=\mathcal{A}(W, 1)$ if and only if $a(d)=n$.

The reader should be warned that no new examples of free or $K(\pi, 1)$ arrangements occur in Theorem 1.3. Nevertheless, the result is essentially all one could hope for and gives a nice extension of the classical picture to arbitrary eigenvalues.

\section{Preliminaries}

Recall that a $G$-poset is a poset $P$ with a $G$-action that preserves order, so that $x<y$ implies $g x<g y$ for all $g \in G$ and $x, y \in P$. It is bounded if it has a unique minimal element (called the bottom element and denoted by $\hat{0}$ ) and a unique maximal element (called the top element and denoted by $\hat{1}$ ). Write $P \cup\{\hat{0}\}$ for the poset obtained from $P$ by adjoining a new element $\hat{0}$, regardless of whether $P$ has a bottom element. Similarly, $P \cup\{\hat{1}\}$ is obtained by adjoining a new element $\hat{1}$. Appending both yields $\widehat{P}:=P \cup\{\hat{0}, \hat{1}\}$. 
The order complex of a poset $P$ is the (abstract) simplicial complex $\Delta(P)$ consisting of all totally ordered sets $x_{1}<x_{2}<\ldots<x_{i}$ in $P$. Because it has a cone point (and is therefore contractible) if $P$ has a top or bottom element, one often considers the proper part $\bar{P}:=P \backslash\{\hat{0}, \hat{1}\}$ of the poset, which is just $P$ if neither a top nor bottom element is present.

Recall that a (finite) simplicial complex $\Delta$ is $k$-connected if its homotopy groups $\pi_{j}(\Delta)$ are trivial for $0 \leq j \leq k$. Define the link of a face $F \in \Delta$ to be the subcomplex

$$
\mathrm{lk}_{\Delta}(F)=\{G \in \Delta: G \cup F \in \Delta, G \cap F=\varnothing\},
$$

and say $\Delta$ is homotopy Cohen-Macaulay (abbreviated to HCM) if for each face $F \in \Delta$ the link $\operatorname{lk}_{\Delta}(F)$ is $\left(\operatorname{dim} \mathrm{lk}_{\Delta}(F)-1\right)$-connected. For $\mathbf{k}$ a field or $\mathbb{Z}$, the simplicial complex is said to be Cohen-Macaulay over $\mathbf{k}$ (abbreviated to $\mathrm{CM} / \mathbf{k}$, or just $\mathrm{CM}$ when $\mathbf{k}=\mathbb{Z})$ if for each face $F \in \Delta$ the homology groups $\widetilde{H}_{i}\left(\operatorname{lk}_{\Delta}(F) ; \mathbf{k}\right)$ vanish for $i<\operatorname{dimlk}_{\Delta}(F)$. The property of being Cohen-Macaulay is invariant under homeomorphisms and implies that the complex is homologically a bouquet of $(\operatorname{dim} \Delta)$-spheres, whereas the stronger property of being homotopy CohenMacaulay is not invariant under homeomorphisms but implies that the complex is homotopically a bouquet of $(\operatorname{dim} \Delta)$-spheres; see [27] and [32, p. 117], respectively, or surveys [4,45].

Though there are many techniques for establishing Cohen-Macaulayness, we shall be concerned with (pure) CL-shellability. A simplicial complex $\Delta$ which is pure $d$-dimensional (i.e., each maximal face under inclusion has dimension $d$ ) is said to be shellable if its maximal faces (called facets) can be ordered $F_{1}, F_{2}, \ldots, F_{\ell}$ so that for each $k$ the subcomplex generated by the first $k$ facets intersects the $(k+1)$ st facet in a pure $(d-1)$-dimensional subcomplex. A poset $P$ is called shellable (resp. HCM, CM) if its order complex $\Delta(P)$ is shellable (resp. HCM, CM). Finally, a $C L$-shellable1 poset is a bounded poset that admits a recursive atom ordering, as defined in Section 4. The following implications for poset shellability are strict:

$$
\text { CL-shellable } \Rightarrow \text { shellable } \Rightarrow \mathrm{HCM} \Rightarrow \mathrm{CM} \Rightarrow \mathrm{CM} / \mathbf{k} \Rightarrow \mathrm{CM} / \mathbb{Q} \text {, }
$$

for any field $\mathbf{k}$ of positive characteristic. Note that a poset $P$ is shellable (resp. HCM, $\mathrm{CM}$ ) if and only if $\bar{P}$, or just $P \backslash\{\hat{1}\}$, is shellable (resp. HCM, CM).

\section{General Reductions}

Shephard and Todd classified all irreducible reflection groups in 34. There are 34 exceptional groups in their classification, labeled $G_{4}, G_{5}, \ldots, G_{37}$, and 3 infinite families, explained below:

- $W\left(A_{n}\right) \subset \mathrm{GL}\left(\mathbb{C}^{n}\right)$.

- $G(m, p, n)$ with $m>1, p$ a divisor of $m$, and $(m, p, n) \neq(2,2,2)$.

- $C_{m}:=G(m, 1,1)$.

$W\left(A_{n}\right)$ denotes the representation of $\mathfrak{S}_{n+1}$ one obtains from the defining representation of 91 after modding out by the fixed space $\mathbb{C}\left(e_{1}+e_{2}+\ldots+e_{n+1}\right)$. For $\boldsymbol{\mu}_{m}$ the set of $m^{\text {th }}$ roots of unity (and $p$ a divisor or $m$ ), the group $G(m, p, n)$ consists of all $n \times n$ monomial matrices with nonzero entries in $\boldsymbol{\mu}_{m}$ whose product lies in $\boldsymbol{\mu}_{m / p}$. General reflection groups decompose into irreducible ones as follows.

\footnotetext{
${ }^{1}$ This recursive formulation of CL-shellability is due to Björner and Wachs 5 .
} 
Proposition 3.1 (Theorem 1.27 in [25]). Let $W \subset \mathrm{GL}(V)$ be a reflection group. Let $V_{1}, \ldots, V_{k}$ denote the nontrivial irreducible submodules of $V$, so that the restriction $W_{i}$ of $W$ to $V_{i}$ is irreducible. Then $W \cong W_{1} \times \cdots \times W_{k}$ and one has an orthogonal sum decomposition $V=V^{W} \oplus V_{1} \oplus \ldots \oplus V_{k}$ with respect to a chosen $W$-invariant positive definite Hermitian form.

Corollary 3.2. Maintain the notation of Proposition 3.1 and let $\zeta$ be a root of unity. Then

$$
E(W, \zeta) \cong E\left(W_{1}, \zeta\right) \times \cdots \times E\left(W_{k}, \zeta\right)
$$

In particular, the family of posets $E(W, \zeta)$ obtained by letting $W$ vary over the three infinite families above is the same as that obtained by letting $W$ vary over the single infinite family $G(m, p, n)$. Another consequence is that Question $\mathrm{A}$ and Theorem 1.2 reduce to irreducible reflection groups.

Corollary 3.3. Maintain the notation of Corollary 3.2. Then the following hold.

(i) $E(W, \zeta)$ is $H C M$ (resp. CM) if and only if each $E\left(W_{i}, \zeta\right)$ is HCM (resp. CM).

(ii) $\widehat{E(W, \zeta)}$ is $C L$-shellable if and only if each $\widehat{E\left(W_{i}, \zeta\right)}$ is $C L$-shellable.

Proof. Note that each $E\left(W_{i}, \zeta\right)$ has a top element $\hat{1}_{i}$. Then (ii) follows from (3) and a homeomorphism of Quillen (see [32, Ex. 8.1] and [46, Thm. 5.1(b)]), while (iii) follows from (3) and [6, Thm. 10.16].

Because of the important role that maximal eigenspaces play in what follows, we make the following convention before proceeding.

Convention 3.4. A maximal $\zeta$-eigenspace for $W$ is one that is not properly contained in any other. Because such a space is minimal with respect to the poset order of $E(W, \zeta)$ given by reverse inclusion, in order to avoid confusion we shall always take minimal and maximal to be with respect to inclusion when dealing with subspaces. For example, " $E \in E(W, \zeta)$ maximal" thus means that $E$ is not properly contained in any $V(g, \zeta)$.

A reflection group $W \subset \mathrm{GL}(V)$ acts on the algebra of polynomial functions $S=S\left(V^{*}\right)$ via $g f(v)=f\left(g^{-1} v\right)$, and Shephard and Todd showed that the subalgebra $S^{W}$ of $W$-fixed polynomials is again polynomial, generated by $n:=\operatorname{dim} V$ algebraically independent homogeneous polynomials $f_{1}, f_{2}, \ldots, f_{n}$, called basic invariants, the degrees of which are uniquely determined by the group $W$ and denoted by $d_{1} \leq d_{2} \leq \ldots \leq d_{n}$. We shall always assume an indexing such that $\operatorname{deg}\left(f_{i}\right)=d_{i}$. For $d>0$, write $a(d)$ for the number of $d_{i}$ divisible by $d$ as in 91 see Springer [35.

Proposition 3.5 (Springer). Let $W$ be a reflection group, let $\zeta$ be a primitive $d^{\text {th }}$ root of unity, and let $f_{1}, f_{2}, \ldots, f_{n}$ be a set of basic invariants for $W$. Set $H_{i}:=\left\{v \in V: f_{i}(v)=0\right\}$. Then one has $\bigcup_{g \in W} V(g, \zeta)=\bigcap_{d \nmid d_{i}} H_{i}$.

As a consequence, the collection of maximal $\zeta$-eigenspaces of $W$ depends only on (the group and) the multiset of degrees $d_{i}$ that are divisible by $d$, which we shall denote by $A(d):=\left\{d_{i}: d \mid d_{i}\right\}$, so that $a(d)=|A(d)|$; see Theorem 7.1 below for a sharper result.

Corollary 3.6. Let $W$ be a reflection group. Let $\zeta$ and $\zeta^{\prime}$ be roots of unity of orders $d$ and $d^{\prime}$ such that $A(d)=A\left(d^{\prime}\right)$. Then the set of maximal $\zeta$-eigenspaces of $W$ coincides with the set of maximal $\zeta^{\prime}$-eigenspaces of $W$. 
Proposition 3.7 (Springer). Let $W$ be a reflection group, let $\zeta$ be a primitive $d^{\text {th }}$ root of unity, and let $E, E^{\prime} \in E(W, \zeta)$ be maximal eigenspaces. Then

(i) $\operatorname{dim} E=a(d)$, and

(ii) $g E=E^{\prime}$ for some $g \in W$.

Corollary 3.8. Let $W$ be a reflection group and let $\zeta$ be a primitive $d^{\text {th }}$ root of unity. Then the following are equivalent:

(i) $E(W, \zeta)=\mathscr{L}_{W}$.

(ii) $\mathbb{C}^{n} \in E(W, \zeta)$.

(iii) $a(d)=n$.

Proof. Clearly (ii) $\Rightarrow$ (ii) $\Leftrightarrow$ (iii). Assume $\mathbb{C}^{n} \in E(W, \zeta)$ so that $\zeta \in W$, and hence $\zeta^{-1} W=W$. Writing $V(g, \zeta)=V\left(\zeta^{-1} g, 1\right)$ for each $g \in W$, it follows that $E(W, \zeta)=\mathscr{L}_{W}$

Theorem 3.9 (Lehrer-Springer [23,24]). Let $W$ be a reflection group, let $\zeta$ be a primitive $d^{\text {th }}$ root of unity, and let $E \in E(W, \zeta)$ be maximal with normalizer

$$
N_{W}(E)=\{g \in W: g E \subseteq E\}
$$

and centralizer

$$
Z_{W}(E)=\{g \in W: g v=v \text { for all } v \in E\} .
$$

Then $\bar{N}:=N_{W}(E) / Z_{W}(E)$ acts as a reflection group on $E$, and the following hold:

(i) If $f_{1}, f_{2}, \ldots, f_{n}$ form a set of basic invariants for $W$, then the restrictions $\left.f_{i}\right|_{E}$ of those whose degree $d_{i}$ is divisible by $d$ form a set of basic invariants for $\bar{N}$.

(ii) The reflecting hyperplanes of $\bar{N}$ on $E$ are the intersections of $E$ with the reflecting hyperplanes of $W$ that do not contain $E$.

(iii) If $W$ is irreducible, then $\bar{N}$ acts irreducibly on $E$.

(iv) $W(d):=\bar{N}$ is uniquely determined by $W$ and $d$, up to conjugation by $W$.

Proposition 3.10. Let $W$ be a reflection group and let $\zeta$ be a root of unity. Then one has an inclusion

$$
E(W, \zeta) \subseteq\left\{E \cap X: X \in \mathscr{L}_{W}, E \in E(W, \zeta) \text { maximal }\right\}
$$

and the following are equivalent:

(i) Equality in (4).

(ii) There exists $E \in E(W, \zeta)$ maximal such that for all $X \in \mathscr{L}_{W}$ one has $E \cap X \in E(W, \zeta)$.

(iii) For every $E \in E(W, \zeta)$ maximal and $X \in \mathscr{L}_{W}$ one has $E \cap X \in E(W, \zeta)$.

(iv) There exists $E \in E(W, \zeta)$ maximal such that $[E, \hat{1}]=\mathscr{L}_{N_{W}(E) / Z_{W}(E)}$.

(v) For every $E \in E(W, \zeta)$ maximal, one has $[E, \hat{1}]=\mathscr{L}_{N_{W}(E) / Z_{W}(E)}$.

Proof. For the inclusion (4), let $Y \in E(W, \zeta)$ and choose a maximal $\zeta$-eigenspace $E$ such that $Y \subseteq E$. Write $E=V(g, \zeta)$ and $Y=V(h, \zeta)$ for some $g, h \in W$. Then $v \in Y$ if and only if $h v=\zeta v=g v$, i.e., if and only if $v \in E \cap V\left(g^{-1} h, 1\right)$. Hence $Y=E \cap X$ for some $X \in \mathscr{L}_{W}$. As for the equivalences, clearly (ii) is equivalent to (iii), which is equivalent to (v) by Theorem 3.9.(ii), and the remaining two equivalences (ii) $\Leftrightarrow$ (iii) and (iv) $\Leftrightarrow($ (v) follow from Proposition 3.7 (ii). 


\section{4. $G(m, p, n)$ CASE OF TheOREM 1.2}

Recall that $\boldsymbol{\mu}_{m}$ denotes the collection of all $m^{\text {th }}$ roots of unity, and that $G(m, p, n)$ denotes the group of all $n \times n$ monomial matrices with nonzero entries in $\boldsymbol{\mu}_{m}$ whose product lies in $\boldsymbol{\mu}_{m / p}$. Note that $G(1,1, n)$ is the defining representation of $\mathfrak{S}_{n}$ given in $\$ 1$, and that $G(1,1, n) \subseteq G(m, p, n) \subseteq G(m, 1, n)$. When $m>p$ the set of reflecting hyperplanes for $G(m, p, n)$ coincides with that for $G(m, 1, n)$ and is given by the union of the following two sets:

$$
\begin{aligned}
& \left\{z_{i}=\xi z_{j}: 1 \leq i<j \leq n, \xi \in \boldsymbol{\mu}_{m}\right\}, \\
& \left\{z_{i}=0: 1 \leq i \leq n\right\} .
\end{aligned}
$$

When $m=p$ the set of reflecting hyperplanes for $G(p, p, n)$ is given by (5).

For roots of unity $\delta_{1}, \delta_{2}, \ldots, \delta_{\ell} \in \boldsymbol{\mu}_{m}$ and an $\ell$-set $\left\{\sigma_{1}, \sigma_{2}, \ldots, \sigma_{\ell}\right\} \subseteq[n]$, identify the 2-line array

$$
\sigma=\left(\begin{array}{cccc}
\sigma_{1} & \sigma_{2} & \ldots & \sigma_{\ell} \\
\delta_{1} \sigma_{2} & \delta_{2} \sigma_{3} & \ldots & \delta_{\ell} \sigma_{1}
\end{array}\right)
$$

with the linear map that fixes each $e_{i}$ with $i \in[n] \backslash\left\{\sigma_{1}, \ldots, \sigma_{\ell}\right\}$ and that sends $e_{\sigma_{i}}$ to $\delta_{i} e_{\sigma_{i+1}}$ for $i \in[\ell-1]$, while $e_{\sigma_{\ell}} \mapsto \delta_{\ell} e_{\sigma_{1}}$. Because multiple arrays may represent the same map, in the next section we will require that $\sigma_{1}<\sigma_{2}, \sigma_{3}, \ldots, \sigma_{\ell}$, but we postpone the restriction until then. Call such an element $\sigma$ a (colored) cycle, and define

- $\ell(\sigma):=\ell$ (the length of $\sigma$ ),

- $\operatorname{Supp}(\sigma):=\left\{\sigma_{1}, \ldots, \sigma_{\ell}\right\}$ (the support of $\sigma$ ), and

- $\operatorname{Col}(\sigma):=\left\{\delta_{1}, \ldots, \delta_{\ell}\right\}$ (the multiset of colors of $\sigma$ ).

With two cycles $\sigma, \sigma^{\prime}$ said to be disjoint if $\operatorname{Supp}(\sigma) \cap \operatorname{Supp}\left(\sigma^{\prime}\right)=\varnothing$, note that any element of $G(m, 1, n)$ can be decomposed into a product $\sigma^{(1)} \sigma^{(2)} \cdots \sigma^{(q)}$ of disjoint cycles, and that such a product is an element of $G(m, p, n)$ if and only if $\prod_{i} \prod_{\delta \in \operatorname{Col}\left(\sigma^{(i)}\right)} \delta$ is an element of $\boldsymbol{\mu}_{m / p}$. The following lemma is a straightforward calculation.

Lemma 4.1. Let $\sigma \in G(m, 1, n)$ be a cycle and write $\sigma=\left(\begin{array}{cccc}\sigma_{1} & \sigma_{2} & \ldots & \sigma_{\ell} \\ \delta_{1} \sigma_{2} & \delta_{2} \sigma_{3} & \ldots & \delta_{\ell} \sigma_{1}\end{array}\right)$. Let $\zeta \neq 1$ be a root of unity. Then

$$
\operatorname{dim} V(\sigma, \zeta)= \begin{cases}1 & \text { if } \zeta^{\ell}=\prod \delta_{i} \\ 0 & \text { otherwise. }\end{cases}
$$

Moreover, in the former case $V(\sigma, \zeta)$ is the solution set of the following equations:

$$
\begin{gathered}
z_{\sigma_{1}}=\zeta \delta_{1}^{-1} z_{\sigma_{2}}=\zeta^{2}\left(\delta_{1} \delta_{2}\right)^{-1} z_{\sigma_{3}}=\ldots=\zeta^{\ell-1}\left(\delta_{1} \delta_{2} \cdots \delta_{\ell-1}\right)^{-1} z_{\sigma_{\ell}}, \\
z_{i}=0 \text { for } i \in[n] \backslash\left\{\sigma_{1}, \sigma_{2}, \ldots, \sigma_{\ell}\right\} .
\end{gathered}
$$

The crux of Theorem 1.2 is Proposition 4.3 below, for which we will need the following.

Lemma 4.2. Let $W=G(m, p, n)$, let $g \in W$, and let $\zeta$ be a primitive $d^{\text {th }}$ root of unity. Suppose that $d \mid m$ and that $a(d)<n$. Then there exists an $i \in[n]$ such that for all $\mathbf{z} \in V(g, \zeta)$ one has $z_{i}=0$. 
Proof. It suffices to assume that $V(g, \zeta)$ is maximal. Since $\zeta \in \boldsymbol{\mu}_{m}$, we have that $W$ contains

$$
h:=\left(\begin{array}{c}
1 \\
\zeta 1
\end{array}\right)\left(\begin{array}{c}
2 \\
\zeta 2
\end{array}\right) \cdots\left(\begin{array}{c}
a(d) \\
\zeta a(d)
\end{array}\right)\left(\begin{array}{c}
n \\
\zeta^{-a(d)} n
\end{array}\right),
$$

which must have $\operatorname{dim} V(h, \zeta)=a(d)$ by Proposition 3.7(1), and therefore $z_{n}=0$ for each $\mathbf{z} \in V(h, \zeta)$. Since $W$ acts transitively on its maximal $\zeta$-eigenspaces by Proposition 3.7(1ii), the result follows.

In the next proposition we define another group $W^{\prime}$ in the $G(m, p, n)$ family that contains $W$ as a subgroup. In particular, $\mathscr{L}_{W} \subset \mathscr{L}_{W^{\prime}}$. We do so to obtain a stronger version of Theorem 1.2 (Theorem 4.4 below) which will be used in Sections 8 and 9 ,

Proposition 4.3. Let $W=G(m, p, n)$, let $\zeta$ be a primitive $d^{\text {th }}$ root of unity, and let $m \vee d$ denote the least common multiple of $m$ and $d$. Set

$$
W^{\prime}:= \begin{cases}W & \text { if } a(d)=n ; \\ G(m \vee d, 1, n) & \text { if } a(d)<n .\end{cases}
$$

Then $V(g, \zeta) \cap X \in E(W, \zeta)$ for every $g \in W$ and $X \in \mathscr{L}_{W^{\prime}}$.

Proof. If $a(d)=n$, then one has $E(W, \zeta)=E(W, 1)=\mathscr{L}_{W}$, and the result follows.

Assume that $a(d)<n$. It suffices to show that $V(g, \zeta) \cap H \in E(W, \zeta)$ for every $g \in W$ and every reflecting hyperplane $H$ of $W^{\prime}$, since a general element of $\mathscr{L}_{W^{\prime}}$ is an intersection of reflecting hyperplanes. Let $g \in W$ and let $r \in W^{\prime}$ be a reflection with fixed space $H$. We show that $V(g, \zeta) \cap H$ is a $\zeta$-eigenspace of $W$ by exhibiting an $h \in W$ such that $V(h, \zeta)=V(g, \zeta) \cap H$.

Set $h=g$ if $V(g, \zeta) \subseteq H$. Assume otherwise so that $V(g, \zeta) \nsubseteq H$. Write $g=\sigma^{(1)} \sigma^{(2)} \cdots \sigma^{(q)}$ as a maximal product of nonempty disjoint cycles so that $[n]=\bigcup_{i} \operatorname{Supp}\left(\sigma^{(i)}\right)$ and $V(g, \zeta)=\bigoplus_{i} V\left(\sigma^{(i)}, \zeta\right)$, and define $\Sigma:=\left\{\sigma^{(1)}, \ldots, \sigma^{(q)}\right\}$. Since $H$ is of the form $z_{j}=0$ or $z_{j}=\xi z_{k}$, we see from Lemma 4.1 that it contains all but exactly one or exactly two of the eigenspaces $V\left(\sigma^{(i)}, \zeta\right)$.

Case 1. There exists exactly one cycle $\sigma \in \Sigma$ such that $V(\sigma, \zeta) \nsubseteq H$.

Subcase 1a. $\ell(\sigma) \geq 2$. Write $\sigma=\left(\begin{array}{ccccc}\sigma_{1} & \sigma_{2} & \ldots & \sigma_{\ell-1} & \sigma_{\ell} \\ \delta_{1} \sigma_{2} & \delta_{2} \sigma_{3} & \ldots & \delta_{\ell-1} \sigma_{\ell} & \delta_{\ell} \sigma_{1}\end{array}\right)$ and let $h$ be the element of $W$ that one obtains from $g$ by replacing $\sigma$ with the product of the cycles

$$
\sigma^{\prime}:=\left(\begin{array}{ccccc}
\sigma_{1} & \sigma_{2} & \ldots & \sigma_{\ell-2} & \sigma_{\ell-1} \\
\delta_{1} \sigma_{2} & \delta_{2} \sigma_{3} & \ldots & \delta_{\ell-2} \sigma_{\ell-1} & \delta_{\ell-1} \delta_{\ell} \sigma_{1}
\end{array}\right) \quad \text { and } \quad \sigma^{\prime \prime}:=\left(\begin{array}{c}
\sigma_{\ell} \\
\sigma_{\ell}
\end{array}\right)
$$

Then $V(h, \zeta)=V(g, \zeta) \cap H$ if and only if $V\left(\sigma^{\prime}, \zeta\right)=V\left(\sigma^{\prime \prime}, \zeta\right)=\{0\}$. Clearly $V\left(\sigma^{\prime \prime}, \zeta\right)=\{0\}$, since $d>1$. Suppose that $V\left(\sigma^{\prime}, \zeta\right) \neq\{0\}$. Since $V(\sigma, \zeta) \neq\{0\}$ also, Lemma 4.1 tells us that $\zeta^{\ell}=\delta_{1} \delta_{2} \cdots \delta_{\ell}=\zeta^{\ell-1}$. However, $\zeta \neq 1$.

For example, if $W=G(2,2,8)$ and $\zeta=e^{2 \pi i / 6}$, then for

$$
\begin{aligned}
g & =\left(\begin{array}{ccc}
1 & 2 & 3 \\
2 & -3 & 1
\end{array}\right)\left(\begin{array}{ccc}
5 & 6 & 7 \\
-6 & -7 & -5
\end{array}\right)\left(\begin{array}{ll}
4 & 8 \\
8 & 4
\end{array}\right), \\
H & =\left\{\mathbf{z} \in \mathbb{C}^{8}: z_{5}=\zeta^{2} z_{6}\right\},
\end{aligned}
$$

one has $h=\left(\begin{array}{ccc}1 & 2 & 3 \\ 2 & -3 & 1\end{array}\right)\left[\left(\begin{array}{cc}5 & 6 \\ -6 & -(-5)\end{array}\right)\left(\begin{array}{l}7 \\ 7\end{array}\right)\right]\left(\begin{array}{ll}4 & 8 \\ 8 & 4\end{array}\right)$. 
Subcase 1b. $\ell(\sigma)=1$. Then $\sigma=\left(\begin{array}{c}\tau_{0} \\ \zeta \tau_{0}\end{array}\right)$ for some $\tau_{0} \in[n]$, implying that $\zeta \in \boldsymbol{\mu}_{m}$, and so $d \mid m$ and $a(d)<n$. It follows from Lemmas 4.1 and 4.2 that $V(\tau, \zeta)=\{0\}$ for some $\tau \in \Sigma$. Note that necessarily $\tau \neq \sigma$, as their $\zeta$-eigenspaces disagree. Write

$$
\tau=\left(\begin{array}{cccc}
\tau_{1} & \tau_{2} & \ldots & \tau_{\ell} \\
\epsilon_{1} \tau_{2} & \epsilon_{2} \tau_{3} & \ldots & \epsilon_{\ell} \tau_{1}
\end{array}\right)
$$

and obtain $h$ from $g$ by replacing the two cycles $\sigma, \tau$ with the single cycle

$$
\sigma^{\prime}=\left(\begin{array}{ccccc}
\tau_{0} & \tau_{1} & \tau_{2} & \ldots & \tau_{\ell} \\
\zeta \tau_{1} & \epsilon_{1} \tau_{2} & \epsilon_{2} \tau_{3} & \ldots & \epsilon_{\ell} \tau_{0}
\end{array}\right)
$$

Applying Lemma 4.1 shows that $V\left(\sigma^{\prime}, \zeta\right)=\{0\}$; indeed, one has $\zeta^{\ell} \neq \prod_{i=1}^{\ell} \epsilon_{i}$, and so $\zeta^{\ell+1} \neq \zeta \prod_{i=1}^{\ell} \epsilon_{i}$. It follows that $V(h, \zeta)=V(g, \zeta) \cap H$. That $h$ is in $W$ is clear.

For example, if $W=G(6,6,4)$ and $\zeta=e^{2 \pi i / 3}$, then for $g=\left(\begin{array}{c}2 \\ \zeta 2\end{array}\right)\left(\begin{array}{c}3 \\ \zeta 3\end{array}\right)\left(\begin{array}{c}4 \\ \zeta 4\end{array}\right)\left(\begin{array}{l}1 \\ 1\end{array}\right)$ and $H=\left\{\mathbf{z} \in \mathbb{C}^{4}: z_{3}=0\right\}$, one has $h=\left(\begin{array}{c}2 \\ \zeta 2\end{array}\right)\left(\begin{array}{c}4 \\ \zeta 4\end{array}\right)\left(\begin{array}{cc}3 & 1 \\ \zeta 1 & 3\end{array}\right)$.

Case 2. There exist exactly two cycles $\sigma, \tau \in \Sigma$ such that $V(\sigma, \zeta), V(\tau, \zeta) \nsubseteq H$.

Then for some $\xi \in \boldsymbol{\mu}_{m \vee d}$ and a suitable indexing, we have $\sigma=\left(\begin{array}{cccc}\sigma_{1} & \sigma_{2} & \ldots & \sigma_{s} \\ \delta_{1} \sigma_{2} & \delta_{2} \sigma_{3} & \ldots & \delta_{s} \sigma_{1}\end{array}\right), \tau=\left(\begin{array}{cccc}\tau_{1} & \tau_{2} & \ldots & \tau_{t} \\ \epsilon_{1} \tau_{2} & \epsilon_{2} \tau_{3} & \ldots & \epsilon_{t} \tau_{1}\end{array}\right), r=\left(\begin{array}{cc}\sigma_{1} & \tau_{1} \\ \xi^{-1} \tau_{1} & \xi \sigma_{1}\end{array}\right)$.

Since $H$ is given by $z_{\sigma_{1}}=\xi z_{\tau_{1}}$, Lemma 4.1 implies that $(V(\sigma, \zeta) \oplus V(\tau, \zeta)) \cap H$ consists of the points $\mathbf{z} \in \mathbb{C}^{n}$ that satisfy $z_{i}=0$ for $i \notin \operatorname{Supp}(\sigma) \cup \operatorname{Supp}(\tau)$ and

$$
\begin{aligned}
z_{\sigma_{1}}=\zeta \delta_{1}^{-1} z_{\sigma_{2}}=\ldots=\zeta^{s-1}\left(\delta_{1} \cdots \delta_{s-1}\right)^{-1} z_{\sigma_{s}} & \\
& =\xi z_{\tau_{1}}=\xi \zeta \epsilon_{1}^{-1} z_{\tau_{2}}=\ldots=\xi \zeta^{t-1}\left(\epsilon_{1} \cdots \epsilon_{t-1}\right)^{-1} z_{\tau_{t}} .
\end{aligned}
$$

Let $k$ be such that the coefficient $\epsilon$ of $z_{\tau_{k}}$ in (9) is an element of $\boldsymbol{\mu}_{m}$. (For existence, note that the cosets $\boldsymbol{\mu}_{m}, \zeta \boldsymbol{\mu}_{m}, \ldots, \zeta^{t-1} \boldsymbol{\mu}_{m}$ cover the group $\boldsymbol{\mu}_{d} \boldsymbol{\mu}_{m}$, since $\zeta$ generates $\boldsymbol{\mu}_{d}$ and $\zeta^{t} \in \boldsymbol{\mu}_{m}$, and then observe that $\xi$ permutes these cosets, since $\boldsymbol{\mu}_{m \vee d}=\boldsymbol{\mu}_{m} \boldsymbol{\mu}_{d}$ from basic algebra.) Then

$$
(V(\sigma, \zeta) \oplus V(\tau, \zeta)) \cap H=(V(\sigma, \zeta) \oplus V(\tau, \zeta)) \cap H^{\prime}
$$

for $H^{\prime}$ the reflecting hyperplane of $r^{\prime}=\left(\begin{array}{cc}\sigma_{1} & \tau_{k} \\ \epsilon^{-1} \tau_{k} & \epsilon \sigma_{1}\end{array}\right)$.

We claim that $h:=g r^{\prime}$ satisfies $V(h, \zeta)=V(g, \zeta) \cap H$, or in other words, that $V\left(\sigma \tau r^{\prime}\right)=V(\sigma \tau) \cap H$. To see this, employ (10) to rewrite the equality as

$$
V\left(\sigma \tau r^{\prime}, \zeta\right)=(V(\sigma, \zeta) \oplus V(\tau, \zeta)) \cap H^{\prime}
$$

Now observe that, on one hand, $V(\sigma \tau, \zeta) \cap H^{\prime}$ is clearly contained in $V\left(\sigma \tau r^{\prime}, \zeta\right)$ and has dimension 1 by hypothesis. On the other hand, $\sigma \tau r^{\prime}$ is necessarily a cycle, and therefore has $\zeta$-eigenspace of dimension at most 1 by Lemma 4.1 . 
For example, if $W=G(6,3,8)$ and $\zeta=e^{2 \pi i / 9}$, then for $\omega:=e^{2 \pi i / 6}$ and

$$
\begin{aligned}
g & =\left(\begin{array}{ccc}
1 & 2 & 3 \\
\omega 2 & 3 & \omega 1
\end{array}\right)\left(\begin{array}{ccc}
4 & 5 & 6 \\
-5 & \omega^{4} 6 & \omega 4
\end{array}\right)\left(\begin{array}{cc}
7 & 8 \\
\omega 8 & \omega 7
\end{array}\right), \\
\xi & =e^{2 \pi i / 18} \\
r & =\left(\begin{array}{cc}
1 & 4 \\
\xi^{-1} 4 & \xi 1
\end{array}\right),
\end{aligned}
$$

one has $r^{\prime}=\left(\begin{array}{cc}1 & 6 \\ \omega^{2} 6 & \omega^{-2} 1\end{array}\right), h=\left(\begin{array}{cccccc}1 & 4 & 5 & 6 & 2 & 3 \\ -4 & -5 & \omega^{4} 6 & \omega^{5} 2 & 3 & \omega 1\end{array}\right)\left(\begin{array}{cc}7 & 8 \\ \omega 8 & \omega 7\end{array}\right)$.

Proof of Theorem 1.2 for $G(m, p, n)$. Note that $\mathscr{L}_{W} \subseteq \mathscr{L}_{W^{\prime}}$ in Proposition 4.3 . since $W$ is a subgroup of $W^{\prime}$, and then invoke Proposition 3.10 .

Another consequence of Proposition 4.3 is the following stronger result, which will play an important role in Sections 8 and 9 below.

Theorem 4.4. Let $W=G(m, p, n)$, let $\zeta$ be a primitive $d^{\text {th }}$ root of unity, and let $W^{\prime}$ be as in Proposition 4.3, Then

(i) $E(W, \zeta) \subseteq \mathscr{L}_{W^{\prime}}$, and

(ii) $V(g, \zeta) \cap X \in E(W, \zeta)$ for every $g \in W$ and $X \in \mathscr{L}_{W^{\prime}}$.

In other words, $E(W, \zeta)$ is an upper order ideal of $\mathscr{L}_{W^{\prime}}$.

Proof. Observe that $W^{\prime}$ contains the scalar matrix $\zeta$, and that

$$
V(g, \zeta)=V\left(\zeta^{-1} g, 1\right) \in \mathscr{L}_{W^{\prime}}
$$

whenever $g \in W$. The first claim follows, and the second is Proposition 4.3 .

\section{Maximal eigenspaces of $G(m, p, n)$}

In this section we associate a certain word, denoted by word $(E)$, to each maximal eigenspace $E \in E(W, \zeta)$ for $W=G(m, p, n)$ and $\zeta \neq 1$. In the next section we show that lexicographically ordering these words gives a recursive atom ordering for $\widehat{E(W, \zeta)}$. In addition to $a(d)$, the following number plays an important role in our discussion:

$$
\begin{aligned}
\ell(d) & \stackrel{\text { def }}{=} \min \left\{s \in \mathbb{Z}_{\geq 1}: \zeta^{s} \in \boldsymbol{\mu}_{m}\right\} \\
& =\frac{d}{\operatorname{gcd}(m, d)} .
\end{aligned}
$$

The crux of our construction is that each maximal eigenspace $E \in E(W, \zeta)$ determines a unique set of $a(d)$ many $\ell(d)$-cycles in $G(m, 1, n)$ whose product has $\zeta$ eigenspace $E$. It is from this set that we construct word $(E)$ in Corollary 5.4 below. We establish the correspondence by first showing that any product $g \in G(m, 1, n)$ of $\operatorname{dim} V(g, \zeta)$ many nontrivial $\ell(d)$-cycles is uniquely determined by its eigenspace $V(g, \zeta)$, and then showing that each maximal $E \in E(W, \zeta)$ may be realized as the $\zeta$-eigenspace of such a product.

Recall from Section 4 the identification of a 2-line array of the form

$$
\sigma=\left(\begin{array}{cccc}
\sigma_{1} & \sigma_{2} & \ldots & \sigma_{\ell} \\
\delta_{1} \sigma_{2} & \delta_{2} \sigma_{3} & \ldots & \delta_{\ell} \sigma_{1}
\end{array}\right)
$$

and a particular element of $G(m, 1, n)$, and note that the element determines the 2line array up to cyclically permuting columns. Thus, by requiring that the smallest 
$\sigma_{i}$ come first, the array is uniquely determined. We adopt this convention for the remainder of the section, i.e., that $\sigma_{1}<\sigma_{2}, \sigma_{3}, \ldots, \sigma_{\ell}$.

Lemma 5.1. Let $\zeta$ be a primitive $d^{\text {th }}$ root of unity for $d>1$, and set $\ell:=\ell(d)$. Suppose that $\sigma, \tau \in G(m, 1, n)$ are two $\ell$-cycles such that $V(\sigma, \zeta)=V(\tau, \zeta) \neq\{0\}$. Then $\sigma=\tau$.

Proof. We show that the map $\sigma \mapsto V(\sigma, \zeta)$ is a bijection when restricted to the $\ell$-cycles $\sigma$ such that $V(\sigma, \zeta) \neq 0$ by constructing its inverse. Fix such a cycle $\sigma$ and label its entries as in (14) so that its image $V(\sigma, \zeta)$ is defined by equations (7) and (8) of Lemma 4.1. Working backwards, first note that $V(\sigma, \zeta)$ is a line, and therefore uniquely determines (7) and (8). Next observe that $\boldsymbol{\mu}_{m \vee d}$ is the disjoint union of the cosets $\boldsymbol{\mu}_{m}, \zeta \boldsymbol{\mu}_{m}, \ldots, \zeta^{\ell} \boldsymbol{\mu}_{m}$, so that for each $i$ there is a unique scalar $\zeta^{j}\left(\delta_{1} \delta_{2} \cdots \delta_{j}\right)^{-1}$ appearing in (7) that is contained in the coset $\zeta^{i} \boldsymbol{\mu}_{m}$, from which one recovers $\delta_{1} \delta_{2} \cdots \delta_{i-1}$ and $\sigma_{i}$. The cycle $\sigma$ is obtained by letting $i$ range from 1 to $\ell$ while taking successive quotients so as to isolate each $\delta_{i}$.

The general case follows:

Proposition 5.2. Let $\zeta$ and $\ell$ be as in Lemma 5.1. Suppose that

$$
S:=\left\{\sigma^{(1)}, \sigma^{(2)}, \ldots, \sigma^{(q)}\right\} \quad \text { and } \quad T:=\left\{\tau^{(1)}, \tau^{(2)}, \ldots, \tau^{(q)}\right\}
$$

are two sets of pairwise disjoint $\ell$-cycles in $G(m, 1, n)$ that satisfy

(1) $\operatorname{dim} V\left(\sigma^{(i)}, \zeta\right)=\operatorname{dim} V\left(\tau^{(i)}, \zeta\right)=1$ for all $i \in[q]$, and

(2) $\bigoplus_{i} V\left(\sigma^{(i)}, \zeta\right)=\bigoplus_{i} V\left(\tau^{(i)}, \zeta\right)$.

Then $S=T$.

Proof. Consider the image of $\bigoplus_{i} V\left(\sigma^{(i)}, \zeta\right)$ under the orthogonal projection of $\mathbb{C}^{n}$ onto $\bigoplus_{t \in \operatorname{Supp}\left(\tau^{(1)}\right)} \mathbb{C} e_{t}$ and employ Lemma 4.1 to show that $V\left(\tau^{(1)}, \zeta\right)$ is equal to $V\left(\sigma^{(k)}, \zeta\right)$ for some $k$. Then apply Lemma 5.1. The result follows by induction.

We now come to the crux of this section.

Proposition 5.3. Let $\zeta$ and $\ell$ be as in Lemma 5.1, and set $W=G(m, p, n)$. Let $E \in E(W, \zeta)$ be maximal under inclusion. Then there exists a set of pairwise disjoint $\ell$-cycles $\left\{\sigma^{(1)}, \ldots, \sigma^{(a(d))}\right\} \subset G(m, 1, n)$ such that

$$
E=V\left(\sigma^{(1)}, \zeta\right) \oplus \ldots \oplus V\left(\sigma^{(a(d))}, \zeta\right) .
$$

Proof. Choose $g \in W$ such that $E=V(g, \zeta)$, and write $g=\tau^{(1)} \tau^{(2)} \cdots \tau^{(q)}$ as a product of disjoint cycles, indexed so that

$$
\operatorname{dim} V\left(\tau^{(i)}, \zeta\right)= \begin{cases}1 & \text { if } 1 \leq i \leq a(d) \\ 0 & \text { otherwise }\end{cases}
$$

Lemma 4.1 tells us that $\ell\left(\tau^{(i)}\right) \geq \ell$ for each $i \in[a(d)]$, and so $n \geq a(d) \ell$. If $n=a(d) \ell$, the claim follows. Assume otherwise so that $n>a(d) \ell$, and set

$$
h=\prod_{i=0}^{a(d)-1}\left(\begin{array}{cccc}
i \ell+1 & i \ell+2 & \ldots & i \ell+\ell \\
\zeta^{\ell}(i \ell+2) & i \ell+3 & \ldots & i \ell+1
\end{array}\right) .
$$

Let $\pi^{\prime}$ be the element of $G(m, 1, n)$ mapping $e_{n}$ to $\zeta^{-a(d) \ell} e_{n}$ while fixing all other $e_{i}$. Clearly $h \pi^{\prime} \in G(m, p, n)$ and

$$
V\left(h \pi^{\prime}, \zeta\right)=V\left(\pi^{(0)}, \zeta\right) \oplus V\left(\pi^{(1)}, \zeta\right) \oplus \ldots \oplus V\left(\pi^{(a(d)-1)}, \zeta\right) \oplus V\left(\pi^{\prime}, \zeta\right)
$$


for

$$
\pi^{(i)}:=\left(\begin{array}{cccc}
i \ell+1 & i \ell+2 & \ldots & i \ell+\ell \\
\zeta^{\ell}(i \ell+2) & i \ell+3 & \ldots & i \ell+1
\end{array}\right) .
$$

Since Lemma 4.1 implies $\operatorname{dim} V\left(\pi^{(i)}, \zeta\right)=1$ for $0 \leq i \leq a(d)-1$, it follows that $V\left(h \pi^{\prime}, \zeta\right)$ is a maximal eigenspace in $E(W, \zeta)$. Hence the result, as $W$ acts transitively on its maximal $\zeta$-eigenspaces by Proposition 3.7(ii).

We can now label each maximal eigenspace of $G(m, p, n)$ by the cycles that it determines. In the next section we shall use this labeling of the maximal eigenspaces to construct a CL-shelling of $\widehat{E(W, \zeta)}$.

Corollary 5.4. Let $\zeta$ and $\ell$ be as in Lemma 5.1, and set $W=G(m, p, n)$. Let $E \in E(W, \zeta)$ be a maximal eigenspace under inclusion. Then there exists a unique sequence

$$
\operatorname{word}(E):=\left(s_{1}, s_{2}, \ldots, s_{n-a(d) \ell} ; \sigma^{(1)}, \ldots, \sigma^{(a(d))}\right)
$$

of integers $s_{i}$ and disjoint $\ell$-cycles $\sigma^{(i)} \in G(m, 1, n)$ with the following properties:

(i) $E=\bigoplus_{i} V\left(\sigma^{(i)}, \zeta\right)$.

(ii) $\left\{s_{1}, s_{2}, \ldots, s_{n-a(d) \ell}\right\}=[n] \backslash \bigcup_{i=1}^{a(d)} \operatorname{Supp}\left(\sigma^{(i)}\right)$.

(iii) $s_{1}<s_{2}<\ldots<s_{n-a(d) \ell}$.

(iv) $\min \operatorname{Supp}\left(\sigma^{(i)}\right)<\min \operatorname{Supp}\left(\sigma^{(j)}\right)$ whenever $i<j$.

Definition 5.5. Linearly order $\boldsymbol{\mu}_{m}$ as follows: set $\delta:=e^{2 \pi i / m}$ and define $\delta^{j}<\delta^{k}$ whenever $0 \leq j<k<m$. Suppose that $\sigma$ and $\tau$ are two distinct cycles of the same length in $G(m, 1, n)$, and let $k$ index the first column in which they differ:

$$
\begin{aligned}
\sigma & =\left(\begin{array}{cccccc}
\sigma_{1} & \sigma_{2} & \ldots & \sigma_{k} & \ldots & \sigma_{\ell} \\
\delta_{1} \sigma_{2} & \delta_{2} \sigma_{3} & \ldots & \delta_{k} \sigma_{k+1} & \ldots & \delta_{\ell} \sigma_{1}
\end{array}\right), \\
\tau & =\left(\begin{array}{cccccc}
\tau_{1} & \tau_{2} & \ldots & \tau_{k} & \ldots & \tau_{\ell} \\
\epsilon_{1} \tau_{2} & \epsilon_{2} \tau_{3} & \ldots & \epsilon_{k} \tau_{k+1} & \ldots & \epsilon_{\ell} \tau_{1}
\end{array}\right) .
\end{aligned}
$$

Define $\sigma<_{\text {lex }} \tau$ if the $k^{\text {th }}$ column of $\sigma$ is lexicographically less than that of $\tau$ in the sense that one of the following holds:

(a) $\sigma_{k}<\tau_{k}$.

(b) $\sigma_{k}=\tau_{k}$ and $\sigma_{k+1}<\tau_{k+1}$.

(c) $\sigma_{k}=\tau_{k}, \sigma_{k+1}=\tau_{k+1}$, and $\delta_{k}<\epsilon_{k}$.

Definition 5.6. Let $W=G(m, p, n)$ and let $\zeta$ be a primitive $d^{\text {th }}$ root of unity for $d>1$. For two words

$$
\begin{aligned}
\operatorname{word}(E) & =\left(s_{1}, \ldots, s_{n-a(d) \ell} ; \sigma^{(1)}, \ldots, \sigma^{(a(d))}\right), \\
\operatorname{word}\left(E^{\prime}\right) & =\left(t_{1}, \ldots, t_{n-a(d) \ell} ; \tau^{(1)}, \ldots, \tau^{(a(d))}\right)
\end{aligned}
$$

of distinct maximal eigenspaces $E, E^{\prime} \in E(W, \zeta)$, define word $(E)<_{\text {lex }} \operatorname{word}\left(E^{\prime}\right)$ if in the first position in which they differ, the term of word $(E)$ is strictly less than the corresponding term of word $\left(E^{\prime}\right)$.

Example 5.7. Ordering the three maximal (-1)-eigenspaces $E_{i}$ of $\mathfrak{S}_{4}$ by their words word $\left(E_{i}\right)$, we have $E_{1}<_{\operatorname{lex}} E_{2}<_{\operatorname{lex}} E_{3}$ for $E_{i}$ the eigenspace labeled by (i) in Figure 2], see Table 1. (Note that $n=a(d) \ell$ in this case.) 
TABLE 1. The maximal (-1)-eigenspaces $E_{i}$ of $\mathfrak{S}_{4}$ in Figure 2 , indexed with respect to lexicographic order on their words.

\begin{tabular}{lcc}
\hline$i$ & $E_{i}=\{\mathbf{z} \in \mathbb{C}$ satisfying $\ldots\}$ & word $\left(E_{i}\right)$ \\
\hline 1 & $z_{1}=-z_{2}, z_{3}=-z_{4}$ & $\left(\left(\begin{array}{ll}1 & 2 \\
2 & 1\end{array}\right),\left(\begin{array}{ll}3 & 4 \\
4 & 3\end{array}\right)\right)$ \\
2 & $z_{1}=-z_{2}, z_{3}=-z_{4}$ & $\left(\left(\begin{array}{ll}1 & 3 \\
3 & 1\end{array}\right),\left(\begin{array}{ll}2 & 4 \\
4 & 2\end{array}\right)\right)$ \\
3 & $z_{1}=-z_{2}, z_{3}=-z_{4}$ & $\left(\left(\begin{array}{ll}1 & 4 \\
4 & 1\end{array}\right),\left(\begin{array}{ll}2 & 3 \\
3 & 2\end{array}\right)\right)$ \\
\hline
\end{tabular}

The maximal spaces in Figures 15 are similarly indexed. In the poset $E\left(\mathfrak{S}_{4}, \zeta\right)$ of Figure 3, for example, the eigenspaces

$$
\begin{aligned}
& E_{3}=\left\{\mathbf{z} \in \mathbb{C}: z_{2}=0, z_{1}=\zeta z_{3}=\zeta^{2} z_{4}\right\}, \\
& E_{5}=\left\{\mathbf{z} \in \mathbb{C}: z_{3}=0, z_{1}=\zeta z_{2}=\zeta^{2} z_{4}\right\}
\end{aligned}
$$

have words

$$
\begin{aligned}
& \operatorname{word}\left(E_{3}\right)=\left(2,\left(\begin{array}{lll}
1 & 3 & 4 \\
3 & 4 & 1
\end{array}\right)\right), \\
& \operatorname{word}\left(E_{5}\right)=\left(3,\left(\begin{array}{lll}
1 & 2 & 4 \\
2 & 4 & 1
\end{array}\right)\right)
\end{aligned}
$$

such that $\operatorname{word}\left(E_{3}\right)<_{\text {lex }} \operatorname{word}\left(E_{5}\right)$.

\section{6. $G(m, p, n)$ CASE OF TheOREM 1.1}

For $P$ a finite graded poset, denote its rank function by $r(x): P \rightarrow \mathbb{Z}$ (with minimal elements having rank 0 ) and its $\operatorname{rank}$ by $r(P):=\max \{r(x): x \in P\}$. Recall that a poset is bounded if it contains both a bottom element $\hat{0}$ and a top element $\hat{1}$, and that an atom in a poset with a $\hat{0}$ is any element that covers $\hat{0}$.

Definition 6.1 (Björner-Wachs [5]). A bounded poset $P$ is said to admit a recursive atom ordering if its $\operatorname{rank} r(P)$ is 1 , or if $r(P)>1$ and there is an ordering of the atoms $a_{1}, \ldots, a_{t}$ that satisfies the following.

(i) Each interval $\left[a_{j}, \hat{1}\right]$ admits a recursive atom ordering in which its atoms that are contained in $\left[a_{i}, \hat{1}\right]$ for some $i<j$ come first.

(ii) If $i<j$ and $a_{i}, a_{j}<x$, then there exists a $k<j$ and an atom $\widetilde{x}$ of $\left[a_{j}, \hat{1}\right]$ for which $a_{k}<\widetilde{x} \leq x$.

A well-known result of Björner and Wachs [5] states that any ordering of the atoms in a totally semimodular poset is a recursive atom ordering. In particular, any ordering of the atoms in a semimodular lattice is a recursive atom ordering, from which the next useful lemma follows immediately.

Lemma 6.2 (Lemma 3 in [33]). If $P$ is a bounded poset in which $[a, \hat{1}]$ is a semimodular lattice for every atom $a \in P$, then an atom ordering $a_{1}, \ldots, a_{t}$ is a recursive atom ordering if and only if it satisfies condition (iii) of Definition 6.1. 
Our goal is to give, when $W=G(m, p, n)$ and $\zeta$ is a root of unity, a recursive atom ordering for $P=\widehat{E(W, \zeta)}$, whose atoms are the maximal $\zeta$-eigenspaces of $W$ under inclusion. By Lemma 6.2, this amounts to producing a candidate ordering and verifying condition (iii) of Definition 6.1. The case $d=1$ is trivial in the sense that there is only one atom. For $d>1$ we order the atoms by their words:

Theorem 6.3. Let $W=G(m, p, n)$ and let $\zeta$ be a primitive $d^{\text {th }}$ root of unity for $d>1$. Then the lexicographic ordering of atoms $E$ of $\widehat{E(W, \zeta)}$ by their words word $(E)$ is a recursive atom ordering.

Proof. By Theorem 1.2 and Lemma 6.2, we need only verify that the atom ordering satisfies condition (iii) of Definition 6.1. The result follows immediately if there is only one atom, so assume otherwise and note that $a(d)<n$ by Corollary 3.8 .

Suppose that $A, B \in E(W, \zeta)$ are two atoms with word $(A)<_{\text {lex }} \operatorname{word}(B)$. Write

$$
\begin{aligned}
& \operatorname{word}(A)=\left(a_{1}, \ldots, a_{n-a(d) \ell} ; \sigma^{(1)}, \ldots, \sigma^{(a(d))}\right), \\
& \operatorname{word}(B)=\left(b_{1}, \ldots, b_{n-a(d) \ell} ; \tau^{(1)}, \ldots, \tau^{(a(d))}\right) .
\end{aligned}
$$

Since any element that lies above both $A$ and $B$ in $E(W, \zeta)$ must be a subspace of $A \cap B$, it suffices to exhibit a maximal eigenspace $C \in E(W, \zeta)$ that satisfies

(i) $\operatorname{word}(C)<_{\text {lex }} \operatorname{word}(B)$, and

(ii) $A \cap B \subseteq B \cap H \subseteq C$ for some hyperplane $H \in \mathscr{L}_{W^{\prime}}$,

where $W^{\prime}$ is as in Proposition 4.3 .

Choose (possibly empty) cycles $\sigma^{(0)}, \tau^{(0)} \in G(m, 1, n)$ such that

$$
\begin{aligned}
g & :=\sigma^{(0)} \sigma^{(1)} \cdots \sigma^{(a(d))}, \\
h & :=\tau^{(0)} \tau^{(1)} \cdots \tau^{(a(d))}
\end{aligned}
$$

are products of disjoint cycles with $g, h \in W$. Then $A=V(g, \zeta)$ and $B=V(h, \zeta)$, since $A=\bigoplus_{i \geq 1} V\left(\sigma^{(i)}, \zeta\right)$ and $B=\bigoplus_{i \geq 1} V\left(\tau^{(i)}, \zeta\right)$ are maximal.

Case $1 . a_{i} \neq b_{i}$ for some $i$. Let $i$ be the smallest such index. Then $a_{i}<b_{i}$ and $a_{i} \in \operatorname{Supp}\left(\tau^{(j)}\right)$ for some $j \geq 1$. Set $C:=r B=V\left(r h r^{-1}, \zeta\right)$ for

$$
r=\left(\begin{array}{ll}
a_{i} & b_{i} \\
b_{i} & a_{i}
\end{array}\right) \text {. }
$$

Since one obtains word $(C)$ by interchanging $a_{i}$ and $b_{i}$ in word $(B)$, it follows that word $(C)<_{\text {lex }}$ word $(B)$. Applying $r$ to both sides of $B \cap H_{r} \subseteq B$ shows that $B \cap H_{r} \subseteq C$. Lastly, $A \cap B \subseteq B \cap H_{r}$ follows from the fact that $z_{a_{i}}=0$ on $A$ and $z_{b_{i}}=0$ on $B$.

For example, if $W=G(4,2,8)$ and $\zeta=e^{2 \pi i / 6}$, then for $\omega:=e^{2 \pi i / 4}$ and

$$
\begin{aligned}
& \operatorname{word}(A)=\left(4,7,\left(\begin{array}{ccc}
1 & 3 & 2 \\
\omega 3 & -2 & \omega 1
\end{array}\right),\left(\begin{array}{ccc}
5 & 6 & 8 \\
-6 & -8 & 5
\end{array}\right)\right), \\
& \operatorname{word}(B)=\left(4,8,\left(\begin{array}{ccc}
1 & 6 & 5 \\
-6 & 5 & -1
\end{array}\right),\left(\begin{array}{ccc}
2 & 7 & 3 \\
7 & 3 & 2
\end{array}\right)\right),
\end{aligned}
$$

one has $r=\left(\begin{array}{ll}7 & 8 \\ 8 & 7\end{array}\right)$ and

$$
\operatorname{word}(C)=\left(4,7,\left(\begin{array}{ccc}
1 & 6 & 5 \\
-6 & 5 & -1
\end{array}\right),\left(\begin{array}{ccc}
2 & 8 & 3 \\
8 & 3 & 2
\end{array}\right)\right) .
$$


Case 2. $a_{i}=b_{i}$ for all $i$. Let $j \geq 1$ be the smallest integer for which $\sigma^{(j)} \neq \tau^{(j)}$. Set $\sigma:=\sigma^{(j)}$ and $\tau:=\tau^{(j)}$, and let $k$ index the first column in which $\sigma$ and $\tau$ differ:

$$
\begin{aligned}
\sigma & =\left(\begin{array}{cccccc}
\sigma_{1} & \sigma_{2} & \ldots & \sigma_{k} & \ldots & \sigma_{\ell} \\
\delta_{1} \sigma_{2} & \delta_{2} \sigma_{3} & \ldots & \delta_{k} \sigma_{k+1} & \ldots & \delta_{\ell} \sigma_{1}
\end{array}\right), \\
\tau & =\left(\begin{array}{cccccc}
\tau_{1} & \tau_{2} & \ldots & \tau_{k} & \ldots & \tau_{\ell} \\
\epsilon_{1} \tau_{2} & \epsilon_{2} \tau_{3} & \ldots & \epsilon_{k} \tau_{k+1} & \ldots & \epsilon_{\ell} \tau_{1}
\end{array}\right) .
\end{aligned}
$$

Note that $\sigma_{i}=\tau_{i}$ for $i \leq k$, and $\delta_{i}=\epsilon_{i}$ for $i<k$.

By Lemma 4.1 each $\mathbf{z} \in A \cap B$ satisfies the two equations

$$
\begin{aligned}
& z_{\sigma_{1}}=\zeta^{k} \delta_{1}^{-1} \cdots \delta_{k}^{-1} z_{\sigma_{k+1}}, \\
& z_{\tau_{1}}=\zeta^{k} \epsilon_{1}^{-1} \cdots \epsilon_{k}^{-1} z_{\tau_{k+1}} .
\end{aligned}
$$

Since $\sigma_{1}=\tau_{1}$ and $\delta_{i}=\epsilon_{i}$ for $i<k$, it follows that

$$
z_{\sigma_{k+1}}=\delta_{k} \epsilon_{k}^{-1} z_{\tau_{k+1}} \quad \text { for all } \quad \mathbf{z} \in A \cap B .
$$

Case 2a. $\sigma_{k+1}<\tau_{k+1}$. Equation (15) says that $A \cap B \subseteq H_{r}$ for the reflection

$$
r=\left(\begin{array}{cc}
\sigma_{k+1} & \tau_{k+1} \\
\epsilon_{k} \delta_{k}^{-1} \tau_{k+1} & \delta_{k} \epsilon_{k}^{-1} \sigma_{k+1}
\end{array}\right)
$$

Set $C:=r B=V\left(r h r^{-1}, \zeta\right)$ so that, as in Case 1 , one has $A \cap B \subseteq B \cap H_{r} \subseteq C$. Then word $(C)<_{\text {lex }} \operatorname{word}(B)$, since $\sigma_{k+1}$ occurs to the right of $\tau_{k+1}$ in $\operatorname{word}(B)$. (More precisely, either $\sigma_{k+1}=\tau_{k+1+i}$ for some $i \geq 1$, or $\sigma_{k+1}$ is in the support of $\tau^{(l)}$ for some $l>j$.)

For example, if $W=G(4,2,9)$ and $\zeta=e^{2 \pi i / 16}$, then for $\omega:=e^{2 \pi i / 4}$ and

$$
\begin{aligned}
\operatorname{word}(A) & =\left(4,\left(\begin{array}{cccc}
1 & 3 & 2 & 7 \\
\omega 3 & -2 & -7 & 1
\end{array}\right),\left(\begin{array}{cccc}
5 & 9 & 6 & 8 \\
9 & \omega 6 & 8 & 5
\end{array}\right)\right) \\
\operatorname{word}(B) & =\left(4,\left(\begin{array}{cccc}
1 & 3 & 9 & 8 \\
\omega 3 & \omega^{-1} 9 & 8 & \omega 1
\end{array}\right),\left(\begin{array}{cccc}
2 & 6 & 5 & 7 \\
\omega^{-1} 6 & 5 & -7 & 2
\end{array}\right)\right),
\end{aligned}
$$

one has $k=2, r=\left(\begin{array}{cc}2 & 9 \\ \omega 9 & \omega^{-1} 2\end{array}\right)$, and

$$
\operatorname{word}(C)=\left(4,\left(\begin{array}{cccc}
1 & 3 & 2 & 8 \\
\omega 3 & -2 & \omega 8 & \omega 1
\end{array}\right),\left(\begin{array}{cccc}
5 & 7 & 9 & 6 \\
-7 & \omega 9 & -6 & 5
\end{array}\right)\right) .
$$

Case 2b. $\sigma_{k+1}=\tau_{k+1}$. Then $\delta_{k}<\epsilon_{k}$. It follows from (15) that $z_{\tau_{k+1}}=0$, and hence $z_{i}=0$ whenever $i \in \operatorname{Supp}(\tau)$, for each $\mathbf{z} \in A \cap B$. In particular, $A \cap B \subseteq B \cap H_{r}=B \cap H_{s}$ for

$$
r=\left(\begin{array}{c}
\tau_{k} \\
\delta_{k} \epsilon_{k}^{-1} \tau_{k}
\end{array}\right) \text { and } s=\left(\begin{array}{c}
\tau_{\ell} \\
\delta_{k}^{-1} \epsilon_{k} \tau_{\ell}
\end{array}\right) .
$$

Set $h^{\prime}:=h r s \in W$ and $C:=V\left(h^{\prime}, \zeta\right)$. First note that $C$ is maximal, since $B$ is maximal and the cycle $\tau r s$ in $h^{\prime}$ has $\operatorname{dim} V(\tau r s, \zeta)=1$ by Lemma 4.1. It is also clear that $B \cap H_{r}=C \cap H_{r}$, and hence $A \cap B \subseteq B \cap H_{r} \subseteq C$. It remains to see that $C \neq B$, from which it follows that $\operatorname{word}(C)<_{\text {lex }} \operatorname{word}(B)$. To this end, observe that $k<\ell$, since $\delta_{i}=\epsilon_{i}$ for $i<k$ and $\delta_{1} \cdots \delta_{\ell}=\zeta^{\ell}=\epsilon_{1} \cdots \epsilon_{\ell}$, while $\delta_{k}<\epsilon_{k}$. It follows that $\tau r s \neq \tau$, and therefore $C \neq B$ by Proposition 5.2 
For example, if $W=G(4,2,8)$ and $\zeta=e^{2 \pi i / 6}$, then for $\omega:=e^{2 \pi i / 4}$ and

$$
\begin{aligned}
& \operatorname{word}(A)=\left(4,7,\left(\begin{array}{ccc}
1 & 3 & 2 \\
\omega 3 & -2 & \omega 1
\end{array}\right),\left(\begin{array}{ccc}
5 & 6 & 8 \\
-6 & -8 & 5
\end{array}\right)\right), \\
& \operatorname{word}(B)=\left(4,7,\left(\begin{array}{ccc}
1 & 3 & 2 \\
-3 & 2 & -1
\end{array}\right),\left(\begin{array}{ccc}
5 & 8 & 6 \\
8 & 6 & 5
\end{array}\right)\right)
\end{aligned}
$$

one has $k=1, r=\left(\begin{array}{c}1 \\ \omega^{-1} 1\end{array}\right), s=\left(\begin{array}{c}2 \\ \omega 2\end{array}\right)$, and

$$
\operatorname{word}(C)=\left(4,7,\left(\begin{array}{ccc}
1 & 3 & 2 \\
\omega 3 & 2 & \omega^{-1} 1
\end{array}\right),\left(\begin{array}{ccc}
5 & 8 & 6 \\
8 & 6 & 5
\end{array}\right)\right) .
$$

\section{Exceptional cases of Theorems 1.1 and 1.2}

Here we establish CL-shellability for exceptional reflection groups, excluding types $E_{6}, E_{7}, E_{8}$. This will conclude our proof of Theorem 1.1. We also finish proving Theorem 1.2 by establishing (11) for all exceptional reflection groups, with no exclusions. Central to all of this is Theorem 7.1 below, which pins down the different posets $E(W, \zeta)$ by characterizing when two eigenvalues give the same poset. Recall that for a reflection group $W$ and positive integer $d$ we write $A(d)$ for the multiset of degrees that are divisible by $d$, so that in particular $a(d)=|A(d)|$ is the dimension of any maximal $\zeta$-eigenspace of the group.

Theorem 7.1. Let $W$ be a reflection group, and let $\zeta$ and $\zeta^{\prime}$ be roots of unity of orders $d$ and $d^{\prime}$, respectively. Then $E(W, \zeta)=E\left(W, \zeta^{\prime}\right)$ if and only if $A(d)=A\left(d^{\prime}\right)$.

We are most interested in the case when $E(W, \zeta)$ has rank at least two and is not $\mathscr{L}_{W}$, so that $a(d) \neq 0,1, n$, where $n$ is the rank of $W$. This is when CL-shellability and (11) are nontrivial. Table 2 lists all instances of an exceptional reflection group and positive integer $d$ such that $a(d) \neq 0,1, n$, so for each $d$ we are interested in $E(W, \zeta)$ for each primitive $d^{\text {th }}$ root of unity $\zeta$. The $d$ 's are grouped according to $A(d)$, so Theorem 7.1 asserts that $E(W, \zeta)=E\left(W, \zeta^{\prime}\right)$ if and only if $d, d^{\prime}$ appear together in Table 2, In other words, Theorem 7.1 reduces the nontrivial exceptional cases of Question $\mathrm{A}$ and (1) to just 23 different posets $E(W, \zeta)$, one for each set of d's in Table 2

Before proving Theorem 7.1, we use general methods to establish CL-shellability and (1) when $d$ is a regular number satisfying $a(d)=2$. These are Corollaries 7.6 and 7.4 below, and with the help of one of them we then prove Theorem 7.1 . Table 2 shows that 14 of its 23 cases satisfy the hypotheses of Corollaries 7.6 and 7.4. For the other 9 cases (Table 3 below) we first outline our basic computer verification of (11) for all of the cases, with no exclusions, and then we outline our basic computer search to find a recursive atom ordering (and thus a CL-shelling) for 5 of the 9 cases. We end by listing the 4 open cases of the strong and weak versions of Question $\mathrm{A}$ involving types $E_{6}, E_{7}, E_{8}$ and various roots of unity, which we write down in Table 4 below.

We need some facts from Springer's theory of regular elements [35, where an element $g$ of a (finite) reflection group $W \subset \mathrm{GL}(V)$ is called $\zeta$-regular if it has a $\zeta$ eigenvector $v$ that is not contained in any reflecting hyperplane for $W$. When such an element $g$ exists, the eigenvalue $\zeta$ is called a regular eigenvalue, and the order $d$ of $\zeta$ is a regular number. For such a number $d$, Springer [35] showed that an element 
TABLE 2. All instances of an exceptional reflection group $W$ of rank $n$ and positive integer $d$ such that $a(d) \neq 0,1, n$. Nonregular cases are indicated by ${ }^{*}$, and values $d, d^{\prime}$ appear together if and only if $A(d)=A\left(d^{\prime}\right)$; see Theorem [7.1. See also Table 10 below.

\begin{tabular}{lllllllll}
\hline$W$ & $a(d)$ & $d$ & & & & & & \\
\hline$G_{25}$ & 2 & 2,6 & $G_{32}$ & 2 & 4,12 & $G_{36}$ & $2^{*}$ & 4 \\
$G_{28}$ & 2 & 3,6 & $G_{33}$ & $2^{*}$ & 4 & & 3 & 3,6 \\
& 2 & 4 & & 3 & 3,6 & $G_{37}$ & 2 & 5,10 \\
$G_{30}$ & 2 & 3,6 & $G_{34}$ & $2^{*}$ & 4,12 & & 2 & 8 \\
& 2 & 4 & $G_{35}$ & 2 & 4 & & 2 & 12 \\
& 2 & 5,10 & & 2 & 6 & & 4 & 3,6 \\
$G_{31}$ & 2 & $3,6,12$ & & 3 & 3 & & 4 & 4 \\
& 2 & 8 & & 4 & 2 & & & \\
\hline
\end{tabular}

$h \in W$ is $\zeta$-regular if and only if $\operatorname{dim} V(h, \zeta)=a(d)$, and by the following result of Springer and Lehrer 24, these regular numbers $d$ of $W$ are easy to compute.

Theorem 7.2 (Lehrer-Springer). For any complex reflection group, a positive integer $d$ is a regular number if and only if it divides as many degrees as it does codegrees.

Lemma 7.3. Let $W$ be a reflection group and let $\zeta$ be a primitive $d^{\text {th }}$ root of unity. Suppose that $d$ is regular and that $E=V(g, \zeta)$ is a maximal $\zeta$-eigenspace of $W$ under inclusion. Then for any reflection $r \in W$ one has $E \cap H_{r}=V(g r, \zeta)$, where $H_{r}:=\operatorname{ker}(1-r)$ denotes the reflecting hyperplane of $r$.

Proof. Suppose that the trivial inclusion $E \cap H_{r} \subseteq V(g r, \zeta)$ is proper. Then by considering dimension, $V(g r, \zeta)$ is necessarily maximal. It follows from a standard argument using Proposition 3.7(iii) and a theorem of Steinberg [39, Thm. 1.5] that $g r$ is therefore conjugate to $g$. In particular, $\operatorname{det}(g r)=\operatorname{det}(g)$. But $\operatorname{det}(r) \neq 1$.

Applying Proposition 3.10(iii) to the case when $\operatorname{dim} E=2$ gives the following.

Corollary 7.4. Maintain the notation and assumptions of Lemma [7.3, and suppose in addition that $a(d)=2$. Then $E(W, \zeta)$ satisfies (11).

Another consequence of Lemma 7.3 is that $E(W, \zeta)$ is connected.

Corollary 7.5. Maintain the notation and assumptions of Lemma 7.3, and suppose in addition that $a(d) \geq 2$. Then (the Hasse diagram of) the poset $E(W, \zeta) \backslash\{\hat{1}\}$ is connected (as a graph). Equivalently, $\Delta(E(W, \zeta) \backslash\{\hat{1}\})$ is connected.

Proof. Consider two maximal eigenspaces $E, E^{\prime} \in E(W, \zeta)$ and choose an element $g \in W$ such that $E^{\prime}=g E$ (possible by Proposition 3.7(iii)). Write $g$ as a product of reflections $r_{k} r_{k-1} \cdots r_{1}$ and define $E_{i}=r_{i} r_{i-1} \cdots r_{1} E$ for each $i$ so that

$$
E=E_{0}, E_{1}, E_{2}, \ldots, E_{k}=E^{\prime}
$$

is a sequence of maximal eigenspaces. Since the intersection of a neighboring pair $E_{i}, E_{i+1}$ is $H_{r_{i+1}} \cap E_{i}$, an element of $E(W, \zeta)$ by Lemma 7.3 , we conclude that $E$ is connected to $E^{\prime}$ in the Hasse diagram of $E(W, \zeta)$, and hence the result. 
Corollary 7.6. Maintain the notation and assumptions of Lemma [7.3, and suppose in addition that $a(d)=2$. Then $\widehat{E(W, \zeta)}$ is $C L$-shellable.

Proof of Theorem 7.1. By Proposition 3.1, we may assume that $W$ is irreducible.

Assume that $A(d) \neq A\left(d^{\prime}\right)$. It is well known [35, Proof of Thm. 3.4(i)] that for any $S \subset[n]$, each irreducible component of $\bigcap_{i \in S} H_{i}$ has dimension $n-|S|$, where recall from Proposition 3.5 that $H_{i}$ is the hypersurface defined by the invariant polynomial $f_{i}$ of a set of basic invariants $f_{1}, f_{2}, \ldots, f_{n}$ whose respective degrees are $d_{1}, d_{2}, \ldots, d_{n}$. In particular,

$$
\bigcap_{d \nmid d_{i}} H_{i} \neq\left(\bigcap_{d \nmid d_{i}} H_{i}\right) \cap\left(\bigcap_{d^{\prime} \nmid d_{i}} H_{i}\right),
$$

for indeed, $n-|A(d)|$ is not equal to $n-\left|A(d) \cup A\left(d^{\prime}\right)\right|$. It follows from (16) that $\bigcap_{d \nmid d_{i}} H_{i} \neq \bigcap_{d^{\prime} \nmid d_{i}} H_{i}$, and so $E(W, \zeta) \neq E\left(W, \zeta^{\prime}\right)$ by Proposition 3.5 .

Assume that $A(d)=A\left(d^{\prime}\right)$ so that $a(d)=a\left(d^{\prime}\right)$, and consider the following cases.

Case 1. Either $W=G(m, p, n)$ or $a(d) \in\{0,1, n\}$. Theorem 1.2 is certainly true when $a(d) \in\{0,1, n\}$, and was established in 4 for $G(m, p, n)$. We thus have

$$
\begin{aligned}
E(W, \zeta) & =\left\{E \cap X: X \in \mathscr{L}_{W} \text { and } E \in E(W, \zeta) \text { maximal }\right\}, \\
E\left(W, \zeta^{\prime}\right) & =\left\{E \cap X: X \in \mathscr{L}_{W} \text { and } E \in E\left(W, \zeta^{\prime}\right) \text { maximal }\right\} .
\end{aligned}
$$

Now observe that the right sides of (17) and (18) agree by Corollary 3.6 .

Case 2. $W$ exceptional, $a(d)=2$, and $d$ regular. In this case (17) and (18) follow from Corollary 17.4, and so again $E(W, \zeta)=E\left(W, \zeta^{\prime}\right)$ by Corollary 3.6.

Case 3. $W$ exceptional, and either $3 \leq a(d) \leq n-1$ or $d$ is not regular. It clearly suffices to assume that $d$ is the smallest (positive) value for which $A(d)=A\left(d^{\prime}\right)$, so that $\zeta$ and $\zeta^{-1}$ are the only primitive $d^{\text {th }}$ roots of unity by Table 2. If $d=d^{\prime}$, then the equality $E(W, \zeta)=E\left(W, \zeta^{\prime}\right)$ follows from the fact that $V(g, \zeta)=V\left(g^{-1}, \zeta^{-1}\right)$. If $d<d^{\prime}$, the classification shows that there exists a prime $p$ such that $\boldsymbol{\mu}_{d^{\prime}}=\boldsymbol{\mu}_{p} \boldsymbol{\mu}_{d}$ and $a(p)=n$. Choose $\zeta_{p}$ of order $p$ and $\zeta_{d}$ of order $d$ such that $\zeta^{\prime}=\zeta_{p} \zeta_{d}$ and note that $\zeta_{p} \in W$ by Proposition 3.8(ii). Since $V\left(g, \zeta_{p} \zeta_{d}\right)=V\left(\zeta_{p}^{-1} g, \zeta_{d}\right)$, it follows that $E\left(W, \zeta^{\prime}\right)=E\left(W, \zeta_{d}\right)$, while $E\left(W, \zeta_{d}\right)=E(W, \zeta)$ from the previous case $d=d^{\prime}$.

TABle 3. Cases in Table 2 when $a(d) \geq 3$ or $d$ is not a regular number.

\begin{tabular}{ll}
\hline$W$ & $\zeta$ \\
\hline$G_{35}$ & -1 \\
$G_{33}, G_{35}, G_{36}, G_{37}$ & $e^{2 \pi i / 3}$ \\
$G_{33}, G_{34}, G_{36}, G_{37}$ & $e^{2 \pi i / 4}$ \\
\hline
\end{tabular}

In 14 of the 23 cases from Table $2, d$ is a regular number satisfying $a(d)=2$, so for these we have CL-shellability and (11) by Corollaries 7.6 and 7.4. The remaining 9 cases of Question A (both versions) and of Theorem 1.2 are listed in Table 3 .

For the 9 remaining cases of Theorem 1.2 listed in Table 3 we used computer algebra software MAGMA to verify (10) in each case by first choosing an element $g \in W$ whose eigenspace $E:=V(g, \zeta)$ is maximal (i.e., of dimension $a(d)$ by

\footnotetext{
${ }^{2}$ For example, $g$ may be taken to be any Coxeter element raised to the power $d_{n} / d$ in each of the regular cases except $W=G_{37}, \zeta=e^{2 \pi i / 4}$ when $d \nmid d_{n}$.
} 
Proposition 3.7(ii) ) and then checking that the set $\left\{E \cap X: X \in \mathscr{L}_{W}\right\}$ is contained in the set

$$
\left\{V\left(g r_{1} \cdots r_{k}, \zeta\right): r_{i} \text { is a reflection of } W \text { and } 0 \leq k \leq a(d)\right\},
$$

from which the desired equality (1) follows by Proposition 3.10(ii).

Regarding the 9 remaining cases of Question A. we first constructed the upper interval $[E, \hat{1}]$ (with $g$ and $E$ as above) and then from the transitivity of $W$ on its maximal $\zeta$-eigenspaces we constructed all such maximal intervals of $E(W, \zeta)$ by taking the $W / N_{W}(E)$-orbit of $[E, \hat{1}]$. When $g$ is regular, we used the additional fact that the normalizer $N_{W}(E)$ is the centralizer $Z_{W}(g):=\{h \in W: h g=g h\}$ of $g$ in $W$. From the collection of these upper intervals, in the three nonregular cases $\left(G_{33}, G_{34}, G_{36}\right.$ with $\zeta=e^{2 \pi i / 4}$ so that $\left.a(d)=2\right)$ we easily verified that $E(W, \zeta)$ is connected, so that $\widehat{E(W, \zeta)}$ is CL-shellable. For the single remaining case of Theorem 1.1 (when $W=G_{33}$ and $\zeta=e^{2 \pi i / 3}$ ) we chose at random one hundred orderings $E=E_{0}, E_{1}, \ldots, E_{40}$ of the maximal eigenspaces (atoms) of $E(W, \zeta)$ with the property that

$$
\operatorname{dist}\left(E, E_{1}\right) \leq \operatorname{dist}\left(E, E_{2}\right) \leq \operatorname{dist}\left(E, E_{3}\right) \leq \ldots \leq \operatorname{dist}\left(E, E_{40}\right),
$$

where $\operatorname{dist}\left(E, E_{i}\right)$ denotes the graph-theoretic distance from $E$ to $E_{i}$ in the restriction of $E(W, \zeta)$ to its bottom two ranks, i.e., to those eigenspaces of dimension either $a(d)$ or $a(d)-1$. We found that each of these orderings was, in fact, a recursive atom ordering. Lastly, we remark that we also found (an unenlightening) recursive atom ordering for $E\left(G_{35},-1\right)$.

TABLE 4. Open cases of the strong and weak versions of Question A.

\begin{tabular}{lll}
\hline$W$ & $\zeta$ \\
\hline$G_{35}\left(\right.$ type $\left.E_{6}\right)$ & $e^{2 \pi i / 3}$ & \\
$G_{36}\left(\right.$ type $\left.E_{7}\right)$ & $e^{2 \pi i / 3}$ & \\
$G_{37}\left(\right.$ type $\left.E_{8}\right)$ & $e^{2 \pi i / 3}$ & $e^{2 \pi i / 4}$ \\
\hline
\end{tabular}

The remaining 4 open cases of the strong and weak versions of Question $\mathrm{A}$ are listed in Table 4. For more evidence, we used Magma to show that $E(W, \zeta)$ is Cohen-Macaulay when $W=G_{35}$ and $\zeta=e^{2 \pi i / 3}$, and we are hopeful that similar computations can be carried out for the remaining groups.

\section{Consequences for the topology of eigenspace arRangements}

Recall that the order complex of a finite poset $P$ is the (abstract) simplicial complex $\Delta(P)$ consisting of all totally ordered sets $x_{1}<x_{2}<\ldots<x_{i}$ in $P$. We adopt the convention of writing $\widetilde{H}_{i}(P)$ for its $i^{\text {th }}$ reduced homology group $\widetilde{H}_{i}(\Delta(P), \mathbb{C})$ and $\widetilde{H}_{i}(x, y)$ for $\widetilde{H}_{i}(\Delta((x, y)), \mathbb{C})$, where $(x, y)$ denotes the open interval formed by $x, y \in P$. If $P$ is a $G$-poset, then $\Delta(P)$ inherits an action of $G$ from $P$, and thus each $\widetilde{H}_{i}(P)$ may be regarded as a $G$-module (i.e., a $\mathbb{C}[G]$-module) by functoriality. One may alternatively consider the module afforded by the $i^{\text {th }}$ reduced cohomology group $\widetilde{H}^{i}(P)=\widetilde{H}^{i}(\Delta(P), \mathbb{C})$, but this representation is just dual to $\widetilde{H}_{i}(P)$. 
Remark 8.1. We adopt the following conventions for the rank and order complex of an empty open interval $I=(x, y)$ and the empty poset $P \backslash\{\hat{1}\}$ when $|P|=1$. If $y$ covers $x$, we set $r(I)=r(P \backslash\{\hat{1}\})=-1$ and take $\Delta(x, y)$ and $\Delta(P \backslash\{\hat{1}\})$ to be the (-1)-dimensional complex $\{\varnothing\}$ containing only the empty face; if $x=y$, we set $r(I)=-2$ and regard $\Delta(x, x)$ as a $(-2)$-dimensional degenerate empty complex $\varnothing$ with no faces at all. Then $\widetilde{H}_{i}(\{\varnothing\})$ is $\mathbb{C}$ when $i=-1$, and 0 otherwise, while we let the reduced homology of $\varnothing$ vanish in all dimensions and define $\widetilde{H}_{-2}(\varnothing):=\mathbb{C}$.

For our purposes, call a finite collection $\mathcal{A}$ of proper complex linear subspaces of $\mathbb{C}^{n}$ an arrangement. Associate with $\mathcal{A}$ its intersection lattice $L(\mathcal{A})$, obtained by ordering all intersections of subspaces in $\mathcal{A}$ by reverse inclusion. Note that the top element $\hat{1}$ of $L(\mathcal{A})$ is the (nonempty) intersection over $\mathcal{A}$, and that the bottom element $\hat{0}$ is $\mathbb{C}^{n}$, the intersection over the empty set. The following celebrated result of Goresky and MacPherson [15] says that the cohomology of the complement $\mathcal{M}_{\mathcal{A}}:=\mathbb{C}^{n} \backslash \bigcup_{X \in \mathcal{A}} X$ is determined by the combinatorial data of the arrangement.

Theorem 8.2 (Goresky-MacPherson). Let $\mathcal{A}$ be a complex arrangement. Then

$$
\widetilde{H}^{i}\left(\mathcal{M}_{\mathcal{A}}\right)=\bigoplus_{x \in L(\mathcal{A}) \backslash\{\hat{0}\}} \widetilde{H}_{2 \operatorname{codim} x-i-2}(\hat{0}, x) \quad \text { for all } i .
$$

Call an arrangement $\mathcal{A}$ a $G$-arrangement if $G \subset \mathrm{GL}\left(\mathbb{C}^{n}\right)$ is such that $g X \in \mathcal{A}$ for every $X \in \mathcal{A}$ and $g \in G$. For such an arrangement both the cohomology of $\mathcal{M}_{\mathcal{A}}$ and the homology of $L(\mathcal{A})$ inherit a $G$-module structure, and Sundaram and Welker [4] established the following equivariant formulation of Theorem 8.2 .

Theorem 8.3 (Sundaram-Welker). For a (finite, complex, linear) G-arrangement $\mathcal{A}$, one has a $G$-module isomorphism

$$
\widetilde{H}^{i}\left(\mathcal{M}_{\mathcal{A}}\right) \cong \bigoplus_{x} \operatorname{Ind}_{G_{x}}^{G} \widetilde{H}_{2 \operatorname{codim} x-i-2}(\hat{0}, x) \quad \text { for all } i,
$$

where $x$ runs over a collection of representatives for the $G$-orbits on $L(\mathcal{A}) \backslash\{\hat{0}\}$, and $G_{x}$ denotes the stabilizer $\{g \in G: g x=x\}$ for $x \in L(\mathcal{A})$.

A consequence of Theorem 1.1. Theorem 4.4. and Theorem 8.3 is that for $W=G(m, p, n)$, one has that the complement $\mathcal{M}(W, d)$ of the proper $\zeta$-eigenspaces of $W$ has torsion-free integral cohomology, and moreover, the top nonvanishing cohomology module of $\mathcal{M}(W, d)$ is equivariantly isomorphic to the top homology module of the proper part of the poset of $\zeta$-eigenspaces:

Proposition 8.4. Let $W$ be an irreducible non-exceptional reflection group of rank $n$. That is, $W$ is either $W\left(A_{n}\right)$ or $G(m, p, n)$ with $m>1$ and $(m, p, n) \neq(2,2,2)$. Let $\zeta$ be a primitive $d^{\text {th }}$ root of unity and let $\mathcal{A}$ be the arrangement of all proper $\zeta$-eigenspaces of $W$, so that $\mathcal{M}_{\mathcal{A}}=\mathcal{M}(W, d)$. Then $L(\mathcal{A})=E(W, \zeta) \cup\left\{\mathbb{C}^{n}\right\}$ and $\mathcal{M}(W, d)$ has torsion-free integral cohomology. Moreover, the following hold:

(i) If $a(d)=n$, so that $\mathbb{C}^{n} \in E(W, \zeta)$, then as $W$-modules

$$
\widetilde{H}^{2 n-a(d)}(\mathcal{M}(W, d)) \cong \widetilde{H}_{a(d)-2}(\overline{E(W, \zeta)}) .
$$

(ii) If $a(d) \neq n$, so that $\mathbb{C}^{n} \notin E(W, \zeta)$, then as $W$-modules

$$
\widetilde{H}^{2 n-a(d)-1}(\mathcal{M}(W, d)) \cong \widetilde{H}_{a(d)-1}(\overline{E(W, \zeta)}) .
$$


Proof. The first claim, that $L(\mathcal{A})=E(W, \zeta) \cup\left\{\mathbb{C}^{n}\right\}$, follows from the fact that $E(W, \zeta)$ is an upper order ideal of an intersection lattice $\mathscr{L}_{W^{\prime}}$; see Theorem 4.4 . Theorem 1.1 implies that all of the homology summands in Theorem 8.2 are free abelian, since shellability is inherited by open intervals. Hence the second claim.

For (iii), suppose that $a(d) \neq n$, so that the intersection lattice $L(\mathcal{A})$ is obtained from $E(W, \zeta)$ by adjoining the new element $\mathbb{C}^{n}$. Because the top element of $L(\mathcal{A})$ has dimension 0 by irreducibility, it follows that the rank of an interval $(\hat{0}, x)$ is equal to $a(d)-\operatorname{dim} x-1$ whenever $x \neq \hat{0}$. Taking $i$ to be $2 n-a(d)-1$, the homology summands $\widetilde{H}_{2 \operatorname{codim} x-i-2}(\hat{0}, x)$ in (19) become $\widetilde{H}_{a(d)-2 \operatorname{dim} x-1}(\hat{0}, x)$. Since the open intervals are shellable, such a summand vanishes if $a(d)-2 \operatorname{dim} x-1$ is not equal to the dimension $a(d)-\operatorname{dim} x-1$ of $\Delta(\hat{0}, x)$, and so the only surviving summand of (19) corresponds to the top element $x=\hat{1}$ of dimension 0 . (ii) is similar.

The first case of Proposition 8.4 is well known, since $E(W, 1)=\mathscr{L}_{W}$ is a geometric lattice. The homology module of $\Delta\left(\overline{\mathscr{L}_{W}}\right)$ for $W=W\left(A_{n}\right)$ has received considerable attention; see Section 1 In this case Stanley 38 used work of Hanlon 18 to express the top homology module, and thus the top cohomology module of $\mathcal{M}\left(W\left(A_{n}\right), 1\right)$, as an induced linear representation. Lehrer and Solomon 22 extended Stanley's results to all cohomology groups of $\mathcal{M}\left(W\left(A_{n}\right), 1\right)$ and conjectured an extension to all (complexified) finite Coxeter groups. In the next section we will extend Stanley's result to arbitrary eigenvalues $\zeta$. We have not explored the possibility of extending Lehrer and Solomon's result.

\section{Combinatorics of $E(W, \zeta)$ In type $A_{n-1}$}

Recall that $E\left(\mathfrak{S}_{n}, 1\right)$ is the well-understood intersection lattice for the braid arrangement. As such, it may be considered as the lattice of all set partitions of $\{1,2, \ldots, n\}$ ordered by refinement. This simple combinatorial model extends to the intersection lattice for $G(r, 1, n)$ through a construction of Dowling [12, which in turn provides a model for $E(W, \zeta)$ via Theorem 4.4 when $W=G(m, p, n)$ and $E(W, \zeta) \neq \mathscr{L}_{W}$. Theorem 4.4 also tells us that the model inherited by $E(W, \zeta)$ is again particularly simple when the minimal elements have a simple description as elements of $\mathscr{L}_{W^{\prime}}$, where $W^{\prime}=G(m \vee d, 1, n)$ and $d$ is the order of $\zeta$. In this section we set $W=\mathfrak{S}_{n}$ and $d>1$. Not only is the resulting combinatorial description for $E\left(\mathfrak{S}_{n}, \zeta\right)$ interesting in its own right, but it naturally distinguishes the poset of $d$-divisible partitions and leads to a precise description of the homotopy type of $E\left(\mathfrak{S}_{n}, \zeta\right) \backslash\{\hat{1}\}$ in terms of integer partitions. Throughout, $d>1$ and $\zeta$ denotes a primitive $d^{\text {th }}$ root of unity.

9.1. Balanced partitions. The purpose of this subsection is to give a combinatorial description of $E\left(\mathfrak{S}_{n}, \zeta\right)$. As outlined above, we do so by viewing $E\left(\mathfrak{S}_{n}, \zeta\right)$ as an upper order ideal of $\mathscr{L}_{W}$ for $W=G(d, 1, n)$, regarded as a Dowling lattice. Though Dowling's original notation [12] is convenient for general groups, the lattice $\mathscr{L}_{W}$ is constructed as a Dowling lattice from the cyclic group $\mathbb{Z} / d \mathbb{Z}$ and lends itself to description in terms of integrally weighted set partitions, a notation that we shall use throughout.

Recall that a set partition of $\{0,1, \ldots, n\}$ is a collection $\pi=\left\{B_{0}, B_{1}, \ldots, B_{\ell}\right\}$ of nonempty disjoint sets with $\bigcup_{i=0}^{\ell} B_{i}=\{0,1, \ldots, n\}$. We call $\ell$ the length of $\pi$ and write $\ell(\pi):=\ell$. Henceforth, we shall assume that the blocks are indexed so that $B_{0}$ is the zero block, i.e., that $0 \in B_{0}$. We also adopt the convention of writing such a 
partition as $B_{0} / B_{1} / \ldots / B_{\ell}$, omitting commas and set braces for individual blocks when working with explicit sets of integers.

From a partition $B_{0} / B_{1} / \ldots / B_{\ell}$ of $\{0,1, \ldots, n\}$ and a positive integer $m$, one obtains an $m$-weighted partition by assigning a weight $w_{i} \in \mathbb{Z} / m \mathbb{Z}$ to each element $i \in B_{1} \cup B_{2} \cup \cdots \cup B_{\ell}$, while elements of $B_{0}$ remain unweighted. Indicating weights with superscripts, we have $015 / 2^{0} 3^{4} / 4^{0}$ and $023 / 1^{2} 5^{2} / 4^{4}$ as examples of weighted partitions for $n=5$, with 023 being the zero block of the latter. We shall also find it useful to depict each weight by an equal number of bars, in which case we superfluously underline the zero block in order to emphasize that its elements bear no weights; see Figures 7 and 8 ,

For $B$ a nonzero block of a weighted partition, let $B^{w}$ denote the block obtained from $B$ by adding $w$ to the weight of each of its elements (modulo $d$ ), and let $B^{\text {erase }}$ denote the block obtained by removing all weights. For example, if $B=1^{3} 3^{0} 4^{4}$ and $d=5$, then $B^{3}=1^{1} 3^{3} 4^{2}$ and $B^{\text {erase }}=134$.

Since $d>1$, the arrangement of reflecting hyperplanes of $G(d, 1, n)$ is given by

$$
\mathcal{A}_{n, d}:=\left\{z_{i}=0: 1 \leq i \leq n\right\} \cup\left\{z_{i}=\zeta^{w} z_{j}: 1 \leq i<j \leq n, w \in \mathbb{Z} / d \mathbb{Z}\right\} .
$$

The intersection lattice $L\left(\mathcal{A}_{n, d}\right)$ is $\mathfrak{S}_{n}$-equivariantly isomorphic (in fact, $G(d, 1, n)$ isomorphic) to the (particular) Dowling lattice $\Pi_{n, d}$ defined as follows:

Definition 9.1. $\Pi_{n, d}$ is the poset of all $\left(d\right.$-)weighted partitions $\pi=B_{0} / \ldots / B_{\ell}$ of $\{0, \ldots, n\}$ with partial order defined by setting $\pi_{1} \leq \pi_{2}$ if for each block $B$ of $\pi_{1}$ there exists a block of $\pi_{2}$ that either contains $B^{\text {erase }}$ or contains $B^{w}$ for some $w$; see Figure 7. It is the Dowling lattice constructed from the cyclic group $\mathbb{Z} / d \mathbb{Z}$; see [12].

We consider $\Pi_{n, d}$ as an $\mathfrak{S}_{n}$-poset by defining $\sigma\left(B_{0} / \ldots / B_{\ell}\right):=\sigma B_{0} / \ldots / \sigma B_{\ell}$, and the above-mentioned equivariant isomorphism $\Pi_{n, d} \stackrel{\sim}{\longrightarrow} L\left(\mathcal{A}_{n, d}\right)$ is given by

$$
B_{0} / \ldots / B_{\ell} \mapsto V\left(B_{0}\right) \oplus \ldots \oplus V\left(B_{\ell}\right),
$$

where $V\left(B_{0}\right):=\mathbf{0}$, and for any nonzero block $B=\left\{b_{1}^{w_{1}}, b_{2}^{w_{2}}, \ldots, b_{k}^{w_{k}}\right\}$ we set

$$
V(B):=\left\{\mathbf{z} \in \mathbb{C}^{n}: \zeta^{w_{1}} z_{b_{1}}=\zeta^{w_{2}} z_{b_{2}}=\ldots=\zeta^{w_{k}} z_{b_{k}} \text { and } z_{i}=0 \text { for } i \notin B\right\} .
$$

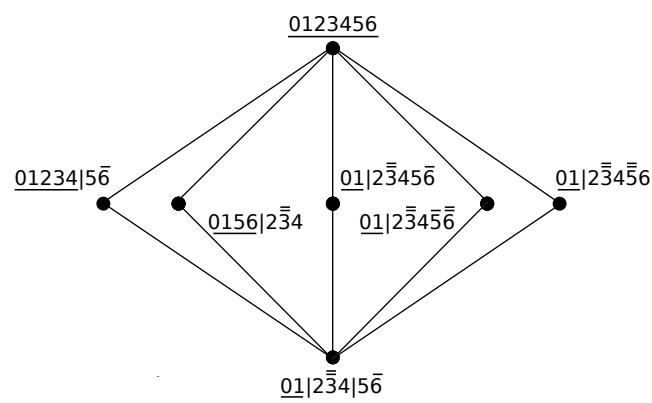

Figure 7. $[x, \hat{1}]$ in $\Pi_{6,3}$ for $x=01\left|2^{0} 3^{2} 4^{0}\right| 5^{0} 6^{1}=01|2 \overline{\overline{3}} 4| 5 \overline{6}$ corresponding to $\left\{\mathbf{z} \in \mathbb{C}^{n}: z_{1}=0, z_{2}=\zeta^{2} z_{3}=z_{4}, z_{5}=\zeta z_{6}\right\}$.

Definition 9.2. Let $d>1$. Call a partition $\pi$ of $\Pi_{n, d}$ balanced if for each block $B$ of $\pi$, all weights $w \in \mathbb{Z} / d \mathbb{Z}$ appear in $B$ with equal multiplicity. The $\mathfrak{S}_{n}$-subposet of $\Pi_{n, d}$ of all balanced partitions is denoted by $\Pi_{n}^{d}$. 


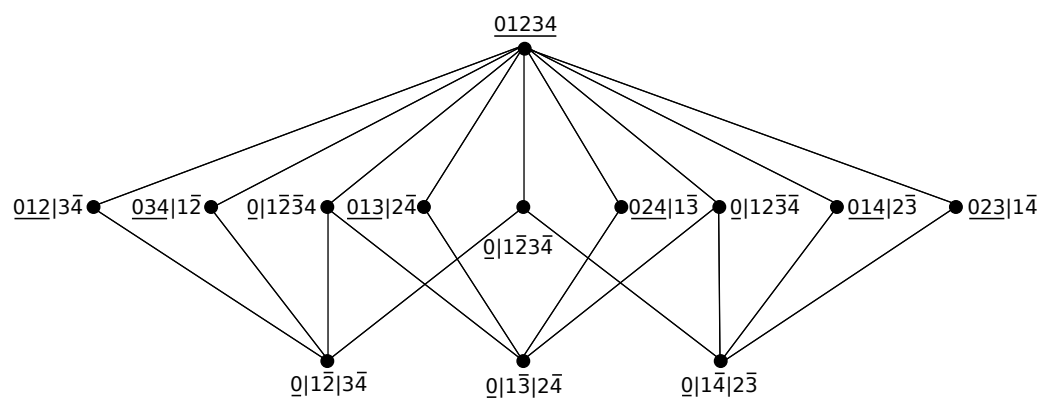

FiguRE $8 . \Pi_{4}^{2}$ with each weight replaced by an equal number of bars.

Recall that an upper order ideal of a poset $P$ is a subposet $I$ with the property that $y \in I$ whenever $x \leq y$ for some $x \in I$. The minimal elements of $I$ are called generators. With this terminology, the next observation follows directly from the definitions of $\Pi_{n, d}$ and $\Pi_{n}^{d}$.

Proposition 9.3. $\Pi_{n}^{d}$ is the upper order ideal of $\Pi_{n, d}$ that is generated by the $\mathfrak{S}_{n}$-orbit of the element $\pi=B_{0} / B_{1} / \ldots / B_{a(d)}$, where $a(d)=\left\lfloor\frac{n}{d}\right\rfloor$ and

$$
B_{0}=\{0\} \cup\{a(d) \cdot d+1, a(d) \cdot d+2, \ldots, n\}
$$

while

$$
\begin{aligned}
B_{1} & =\left\{1^{0}, 2^{1}, \ldots, d^{d-1}\right\}, \\
B_{2} & =\left\{(d+1)^{0},(d+2)^{1}, \ldots,(2 d)^{d-1}\right\}, \\
& \vdots \\
B_{a(d)} & =\left\{((a(d)-1) \cdot d+1)^{0}, \ldots,(a(d) \cdot d)^{d-1}\right\} .
\end{aligned}
$$

Remark 9.4. Note that $r\left(\Pi_{n}^{d}\right)=a(d)$ and $\ell(x)=r([x, \hat{1}])$ for $x \in \Pi_{n}^{d}$. Also observe that $\Pi_{n}^{d}$ has a bottom element $\hat{0}$ if and only if $n=d=2$ or $d>n$. In the latter case, the top and bottom elements coincide, so that $\Pi_{n}^{d}$ is the trivial poset consisting of the element $01 \cdots n$.

Theorem 9.5. Let $d>1$. Then $E\left(\mathfrak{S}_{n}, \zeta\right) \cong \Pi_{n}^{d}$ as $\mathfrak{S}_{n}$-posets.

Proof. Because $\Pi_{n}^{d}$ and $E\left(\mathfrak{S}_{n}, \zeta\right)$ are upper order ideals of $\Pi_{n, d}$ and $L\left(\mathcal{A}_{n, d}\right)$, respectively, it suffices to show that the minimal elements of $\Pi_{n}^{d}$ correspond to those of $E\left(\mathfrak{S}_{n}, \zeta\right)$ under the isomorphism $\Pi_{n, d} \rightarrow L\left(\mathcal{A}_{n, d}\right)$ given in (20). In fact, since the isomorphism is equivariant, by transitivity it suffices to see that the distinguished minimal element of $\Pi_{n}^{d}$ in Proposition 9.3 corresponds to some maximal $\zeta$-eigenspace of $\mathfrak{S}_{n}$. But this is clear, for the distinguished element corresponds to the $\zeta$-eigenspace of

$(1,2, \ldots, d)(d+1, d+2, \ldots, 2 d) \cdots((a(d)-1) d+1,(a(d)-1) d+2, \ldots, a(d) d)$, which is maximal under inclusion by considering dimension.

A general feature of Dowling lattices is that their upper intervals $[x, \hat{1}]$ are again Dowling lattices; see [12, Thm. 2]. For $x \in \Pi_{n, d}$ one can see this by writing $x=B_{0} / B_{1} / \ldots / B_{\ell}$ and then constructing a poset isomorphism $[x, \hat{1}] \rightarrow \Pi_{\ell(x), d}$ by 
mapping any block of the form $B_{i_{1}}^{w_{1}} \cup \cdots \cup B_{i_{j}}^{w_{j}}$ with $w_{i} \in \mathbb{Z} / d \mathbb{Z}$ to $\left\{i_{1}^{w_{1}}, \ldots, i_{j}^{w_{j}}\right\}$ and mapping any zero block of the form $B_{0} \cup B_{i_{1}}^{\text {erase }} \cup \cdots \cup B_{i_{j}}^{\text {erase }}$ to zero block $\left\{0, i_{1}, \ldots, i_{j}\right\}$.

Lemma 9.6. $[x, \hat{1}] \cong \Pi_{\ell(x), d}$ for each $x \in \Pi_{n}^{d}$.

9.2. The topology of balanced partitions. Recall that $E\left(\mathfrak{S}_{n}, \zeta\right) \backslash\{\hat{1}\}$ being shellable implies that its order complex is homotopy equivalent to a bouquet of spheres. The aim of this subsection is to calculate the exact number of such spheres. Central to our analysis is the following consequence of the Hopf trace formula.

Theorem 9.7 (Sundaram [40]). For P a Cohen-Macaulay G-poset with top element $\hat{1}$, one has an isomorphism of virtual $G$-modules

$$
\widetilde{H}_{r(P)-1}(P \backslash\{\hat{1}\}) \cong \bigoplus_{i=0}^{r(P)}(-1)^{r(P)+i} \bigoplus_{x^{G} \in P / G} \operatorname{Ind}_{G_{x}}^{G} \widetilde{H}_{i-2}(x, \hat{1}) .
$$

We shall also need the following consequence of Dowling's well-known description of the Möbius function for any Dowling lattice [12] and Lemma 9.6 .

Lemma 9.8. Let $x \in \Pi_{n}^{d}$. Then $\operatorname{dim} \widetilde{H}_{\ell(x)-2}(x, \hat{1})=\prod_{i=0}^{\ell(x)-1}(1+i d)$.

Regard an integer partition $\lambda$ of $n \geq 0$ as a multiset of positive integers $\lambda_{1}, \ldots, \lambda_{\ell}$ with $\sum \lambda_{i}=n$, and write $|\lambda|=n$ or $\lambda \vdash n$. The integer $\ell(\lambda):=\ell$ is the length of $\lambda$. We shall also regard the partition $\lambda$ as a sequence $\lambda=\left(\lambda_{1}^{m_{1}}, \ldots, \lambda_{p}^{m_{p}}\right)$ of distinct positive integers $0<\lambda_{1}<\lambda_{2}<\ldots<\lambda_{p}$ with positive weights $m_{i}$ satisfying $\sum_{i=1}^{p} m_{i} \lambda_{i}=n$. The integer $p(\lambda):=p$ is the number of distinct parts of $\lambda$. In what follows, the convention being used will be made clear by the presence or absence of weights $m_{i}$.

A pointed partition $\Lambda=\left(\lambda_{0}, \lambda\right)$ of $n \geq 0$ is a nonnegative integer $0 \leq \lambda_{0} \leq n$ together with a partition $\lambda$ of $n-\lambda_{0}$. Such a partition naturally arises in Dowling lattices as the type of a partition $x=B_{0} / B_{1} / \ldots / B_{\ell}$, defined to be

$$
\operatorname{type}(x) \stackrel{\text { def }}{=}\left(\left|B_{0} \backslash\{0\}\right|,\left\{\left|B_{1}\right|, \ldots,\left|B_{\ell}\right|\right\}\right) .
$$

Corollary 9.9. $\Delta\left(\Pi_{n}^{d} \backslash\{\hat{1}\}\right)$ is homotopy equivalent to a bouquet of

$$
\sum_{0 \leq|\lambda| \leq a(d)} \frac{(-1)^{a(d)+\ell(\lambda)}}{d^{\ell(\lambda)}(n-d|\lambda|) !} \frac{n !}{\prod_{j=1}^{p} \lambda_{j} !^{m_{j} d} m_{j} !} \prod_{i=0}^{\ell(\lambda)-1}(1+i d)
$$

many $(a(d)-1)$-spheres.

Proof. Start by noting that two partitions $x, y \in \Pi_{n}^{d}$ are in the same $\mathfrak{S}_{n}$-orbit if and only if $\operatorname{type}(x)=\operatorname{type}(y)$, and observe that for type $(x)=\left(\lambda_{0}, \lambda\right)$ one has

$$
\left|\operatorname{Stab}_{\mathfrak{S}_{n}}(x)\right|=d^{\ell(\lambda)} \lambda_{0} ! \prod_{i=1}^{p} m_{i} !\left(\lambda_{i} / d\right) !^{m_{i} d}
$$

Taking dimensions in Theorem 9.7 and employing (22) and Lemma 9.8 gives the result.

We list some initial values of (21) in Table 5 . 
TABLE $5 . \operatorname{dim} \widetilde{H}_{a(d)-1}\left(\Pi_{n}^{d} \backslash\{\hat{1}\}\right)$ for $n \leq 9$ and $a(d) \neq 0$.

\begin{tabular}{lllllllll}
\hline$n \backslash d$ & 2 & 3 & 4 & 5 & 6 & 7 & 8 & 9 \\
\hline 2 & 0 & & & & & & & \\
3 & 2 & 1 & & & & & & \\
4 & 1 & 7 & 5 & & & & & \\
5 & 21 & 19 & 29 & 23 & & & & \\
6 & 24 & 91 & 89 & 143 & 119 & & & \\
7 & 510 & 841 & 209 & 503 & 839 & 719 & & \\
8 & 918 & 3529 & 5251 & 1343 & 3359 & 5759 & 5039 & \\
9 & 22246 & 32367 & 50275 & 3023 & 10079 & 25919 & 45359 & 40319 \\
\hline
\end{tabular}

Remark 9.10. One may identify the stabilizer $\operatorname{Stab}_{\mathfrak{S}_{n}}(x)$ of $x=B_{0} / B_{1} / \ldots / B_{\ell}$ as a (suitably defined) product of nested wreath products

$$
\mathfrak{S}_{\lambda_{0}} \times \mathfrak{S}_{m_{1}}\left[C_{d}\right]\left[\mathfrak{S}_{\lambda_{1} / d}\right] \times \ldots \times \mathfrak{S}_{m_{p}}\left[C_{d}\right]\left[\mathfrak{S}_{\lambda_{p} / d}\right],
$$

from which one can show that

$$
\widetilde{H}_{\ell-2}(x, \hat{1}) \cong_{\operatorname{Stab}_{\mathfrak{S}_{n}}(x)} \operatorname{Res}_{\operatorname{Stab}_{\mathfrak{S}_{n}}(x)}^{\mathfrak{S}_{\lambda_{0}} \times \mathfrak{S}_{\ell}\left[C_{d}\right]\left[\mathfrak{S}_{n}\right]} \mathbb{1} \otimes \widetilde{H}_{\ell-2}\left(\bar{\Pi}_{\ell, d}\right)[\mathbb{1}]
$$

for a suitable embedding $\operatorname{Stab}_{\mathfrak{S}_{n}}(x) \subseteq \mathfrak{S}_{\lambda_{0}} \times \mathfrak{S}_{\ell}\left[C_{d}\right]\left[\mathfrak{S}_{n}\right]$. Here, $\widetilde{H}_{\ell-2}\left(\bar{\Pi}_{\ell, d}\right)[\mathbb{1}]$ denotes the $\mathfrak{S}_{\ell}\left[C_{d}\right]\left[\mathfrak{S}_{n}\right]$-module $\mathbb{1}^{\otimes \ell d} \otimes \widetilde{H}_{\ell-2}\left(\bar{\Pi}_{\ell, d}\right)$ given by

$$
\left(\sigma_{1}, \ldots, \sigma_{\ell d} ; \sigma\right)\left(v_{1} \otimes \cdots \otimes v_{n} \otimes w\right)=v_{1} \otimes \cdots \otimes v_{n} \otimes \sigma w
$$

for $\sigma_{i} \in \mathfrak{S}_{n}$ and $\sigma \in \mathfrak{S}_{\ell}\left[C_{d}\right]$, and $\Pi_{\ell, d}$ is viewed as a $\mathfrak{S}_{\ell}\left[C_{d}\right]$-poset. Employing Theorem 9.7. one then obtains a (virtual) expression for $\widetilde{H}_{a(d)-1}\left(\Pi_{n}^{d} \backslash\{\hat{1}\}\right)$ as an $\mathfrak{S}_{n}$-module in terms of the $\mathfrak{S}_{\ell}\left[C_{d}\right]$-modules $\widetilde{H}_{\ell-2}\left(\bar{\Pi}_{\ell, d}\right)$ studied by Hanlon [18] and Gottlieb-Wachs [16].

Problem 9.11. Describe the $\mathfrak{S}_{n}$-irreducible decomposition of $\widetilde{H}_{a(d)-1}\left(\Pi_{n}^{d} \backslash\{\hat{1}\}\right)$ explicitly in general.

Tables 6] give the decomposition of $\widetilde{H}_{a(d)-1}\left(\Pi_{n}^{d} \backslash\{\hat{1}\}\right)$ into irreducible submodules for $n=4,5,6,7$. Each entry lists the multiplicity with which the Specht module $S^{\lambda}$ occurs in $\widetilde{H}_{a(d)-1}\left(\Pi_{|\lambda|}^{d} \backslash\{\hat{1}\}\right)$.

TABLE 6. Decomposition of the $\mathfrak{S}_{4}$-module $\widetilde{H}_{a(d)-1}\left(\Pi_{4}^{d} \backslash\{\hat{1}\}\right)$.

\begin{tabular}{lllll}
\hline$d \backslash \lambda$ & 甲 & $\boxplus$ & 甲 & 日 \\
\hline 2 & & & & 1 \\
3 & 1 & & 1 & 1 \\
4 & & 1 & 1 & \\
\hline
\end{tabular}


TABLE 7. Decomposition of the $\mathfrak{S}_{5}$-module $\widetilde{H}_{a(d)-1}\left(\Pi_{5}^{d} \backslash\{\hat{1}\}\right)$.

\begin{tabular}{lcccccc}
\hline$d \backslash \lambda$ & ए & $\boxplus$ & $\boxminus$ & $\Xi$ & 目 & 日 \\
\hline 2 & & 1 & 1 & 1 & 1 & 1 \\
3 & 1 & 1 & 1 & & 1 & \\
4 & 1 & 1 & 1 & 2 & 1 & \\
5 & & 1 & 2 & 1 & & 1 \\
\hline
\end{tabular}

TABLE 8. Decomposition of the $\mathfrak{S}_{6}$-module $\widetilde{H}_{a(d)-1}\left(\Pi_{6}^{d} \backslash\{\hat{1}\}\right)$.

\begin{tabular}{|c|c|c|c|c|c|c|c|c|c|c|}
\hline$d \backslash \lambda$ & سست & $\boxplus$ & $\theta^{m}$ & $\boxplus$ & $\boxplus$ & E & $\boxplus$ & $\boxplus$ & 目 & 目 \\
\hline 2 & & & & & & 1 & & 1 & 1 & \\
\hline 3 & & & 1 & & 2 & 2 & 1 & 2 & 1 & 1 \\
\hline 4 & 1 & 2 & 1 & & 2 & 1 & 1 & 1 & & \\
\hline 5 & 1 & 1 & 2 & 1 & 4 & 2 & 1 & 1 & 1 & 1 \\
\hline 6 & & 2 & 2 & 1 & 2 & 2 & 2 & 1 & 1 & \\
\hline
\end{tabular}

TABLE 9. Decomposition of the $\mathfrak{S}_{7}$-module $\widetilde{H}_{a(d)-1}\left(\Pi_{7}^{d} \backslash\{\hat{1}\}\right)$.

\begin{tabular}{|c|c|c|c|c|c|c|c|c|c|c|c|c|c|c|}
\hline$d \backslash \lambda$ & ס्माप & Шण & बשי & $\boxplus$ & $\Xi^{\square}$ & ש & $\boxplus$ & $\boxplus$ & $\boxplus$ & 百 & $\boxplus$ & 甲 & 目 & \\
\hline 2 & & & & 1 & 2 & 2 & 2 & 2 & 5 & 3 & 2 & 3 & 2 & \\
\hline 3 & & 1 & 2 & 1 & 5 & 4 & 3 & 3 & 7 & 4 & 3 & 3 & 2 & 1 \\
\hline 4 & 1 & 2 & 1 & 1 & 2 & 1 & & 1 & 1 & & & & & \\
\hline 5 & 1 & 2 & 2 & 1 & 4 & 2 & 3 & 2 & 3 & 1 & 1 & & 1 & \\
\hline 6 & 1 & 2 & 2 & 3 & 6 & 4 & 3 & 4 & 5 & 3 & 3 & 2 & 1 & \\
\hline 7 & & 2 & 3 & 2 & 5 & 2 & 3 & 3 & 5 & 3 & 2 & 2 & & 1 \\
\hline
\end{tabular}

9.3. The $\boldsymbol{d}$-divisible partition poset. Throughout this section we shall assume that $d>1$. For $n+1$ divisible by $d$, recall that a set partition of $\{1,2, \ldots, n+1\}$ is called $d$-divisible if all blocks have size divisible by $d$, and let $P_{n+1}^{d}$ denote the $\mathfrak{S}_{n+1^{-}}$ poset of all such partitions ordered by refinement. The $d$-divisible partition lattice $P_{n+1}^{d} \cup\{\hat{0}\}$ has been extensively studied, starting with Sylvester 42 computing the Möbius function for $d=2$. Stanley [37] generalized Sylvester's result to arbitrary $d$ and conjectured that the restriction of the top (reduced) homology module of $P_{n+1}^{d} \backslash\{\hat{1}\}$ to $\mathfrak{S}_{n}$ is the Specht module of skew ribbon shape $(d-1, d, d, \ldots, d)$; see Figure 9. (One instead obtains the regular representation of $\mathfrak{S}_{n}$ if $d=1$; see [38.) Calderbank-Hanlon-Robinson 9] established Stanley's conjecture via character calculations, and Wachs 44] later exhibited an explicit basis for the top homology group via an EL-shelling. Ehrenborg and Jung [13] extended this phenomenon to more general posets of pointed partitions $\Pi_{\vec{c}}^{\bullet}$, showing that each $\Pi_{\vec{c}}^{\bullet} \backslash\{\hat{1}\}$ 
is either contractible or has top homology the $\mathfrak{S}_{n}$-Specht module of skew ribbon shape associated with the composition $\vec{c}=\left(c_{1}, c_{2}, \ldots, c_{k}\right)$.

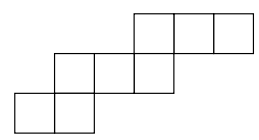

Figure 9. Skew ribbon shape $(2,3,3)$.

In this section we present an intermediate family of posets $\Pi_{n}^{(d)}$, between the family of $d$-divisible partition posets $P_{n+1}^{d}$ and the family of pointed partition posets $\Pi_{\vec{c}}^{\bullet}$, that arises naturally in the context of eigenspace arrangements for $\mathfrak{S}_{n}$. Furthermore, we conjecture that the top homology of $\Pi_{n}^{(d)} \backslash\{\hat{1}\}$ is isomorphic to a submodule of the top homology of $\Pi_{n}^{d} \backslash\{\hat{1}\}$.

For a pointed partition $\Lambda=\left(\lambda_{0}, \lambda\right)$ of $n$, let $\Pi_{\Lambda} \subseteq \Pi_{n, 1}$ denote the upper order ideal generated by the elements of type $\Lambda$ and regard it as an $\mathfrak{S}_{n}$-poset through the usual action of $\mathfrak{S}_{n}$ on $[n]$. For the particular choice

$$
\Lambda=(n-d \cdot a(d),\{d, d, \ldots, d\}),
$$

write $\Pi_{n}^{(d)}$ for $\Pi_{\Lambda}$ and call it the pointed d-divisible partition poset. It is $\mathfrak{S}_{n^{-}}$ isomorphic to Ehrenborg and Jung's poset $\Pi_{(d, d, \ldots, d, n-d \cdot a(d))}^{\bullet^{\prime}}$ via the map that removes all weights and then replaces each zero block $B_{0}$ with the distinguished block $Z:=B_{0} \backslash\{0\}$; see [13] for details. For $n+1$ divisible by $d$, the map

$$
0 \mapsto n+1 \quad \text { and } \quad B \mapsto B^{\text {erase }} \text { for each nonzero block } B
$$

extends to an $\mathfrak{S}_{n}$-isomorphism $\Pi_{n}^{(d)} \rightarrow P_{n+1}^{d}$ when $P_{n+1}^{d}$ is considered as an $\mathfrak{S}_{n^{-}}$ poset by restricting its natural $\mathfrak{S}_{n+1}$-action to $\mathfrak{S}_{\{1, \ldots, n\}}$. As a consequence of this discussion and the main results of 13 we have the following.

Theorem 9.12 (Calderbank-Hanlon-Robinson, Ehrenborg-Jung, Wachs). $\widetilde{H}_{a(d)-1}\left(\Pi_{n}^{(d)} \backslash\{\hat{1}\}\right)$ is isomorphic to the $\mathfrak{S}_{n}$-Specht module of skew ribbon shape associated with $(n-d \cdot a(d), d, d, \ldots, d)$ when $d \nmid n$, and 0 otherwise.

Remark 9.13. We have stated Theorem 9.12 in a way that requires the observation that the skew shapes associated to $(n-d \cdot a(d), d, d, \ldots, d)$ and its reverse $(d, d, \ldots, d, n-d \cdot a(d))$ are related by $180^{\circ}$ rotation, and hence their Specht modules are $\mathfrak{S}_{n}$-isomorphic; see [36, Exercise 7.56].

Note that the $\mathfrak{S}_{n}$-ribbon representation of Theorem 9.12 arises from a subarrangement of the reflection arrangement of $\mathfrak{S}_{n+1}$ :

$$
\Pi_{n}^{(d)} \subseteq \Pi_{n, 1} \cong_{\mathfrak{S}_{n}} \Pi_{n+1} \cong_{\mathfrak{S}_{n}} E\left(\mathfrak{S}_{n+1}, 1\right) \quad \text { with } \quad \mathfrak{S}_{n}=\mathfrak{S}_{\{1, \ldots, n\}},
$$

where the isomorphism $\Pi_{n, 1} \cong_{\mathfrak{S}_{n}} \Pi_{n+1}$ is obtained by extending (23); see Figure 10. We conjecture that these $\mathfrak{S}_{n}$-ribbon representations also appear in $E\left(\mathfrak{S}_{n}, \zeta_{d}\right)$, without the (a priori) mysterious shift of index. To make the assertion more precise, we introduce the new operation $B \mapsto B^{\text {zero }}$ of zeroing: given a block $B$, define $B^{\text {zero }}$ to be the block obtained from $B$ by replacing each weight $w$ with 0 . An immediate observation is the following; see Figure 11 

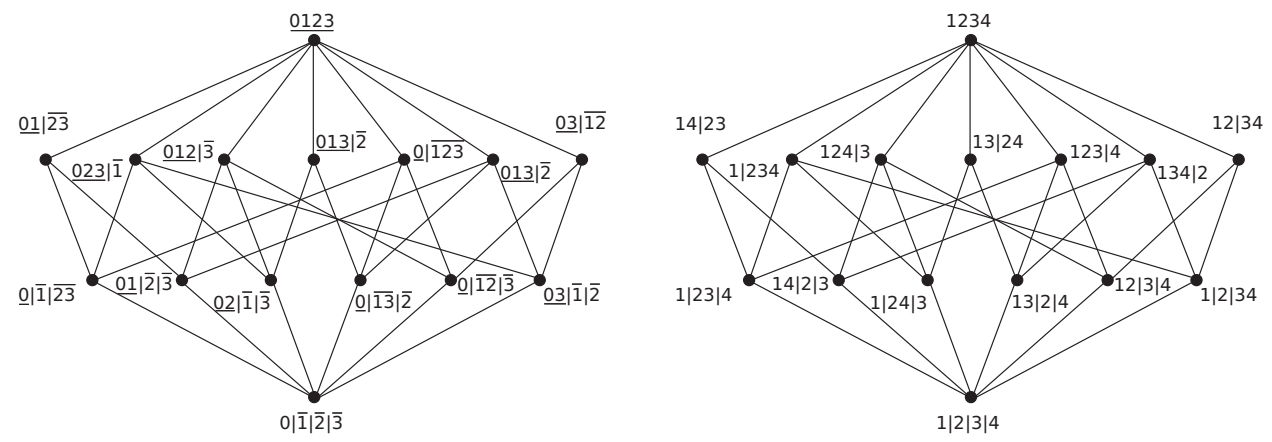

FiguRE 10. The posets $\Pi_{n, 1}$ and $\Pi_{n+1}$ when $n=3$.
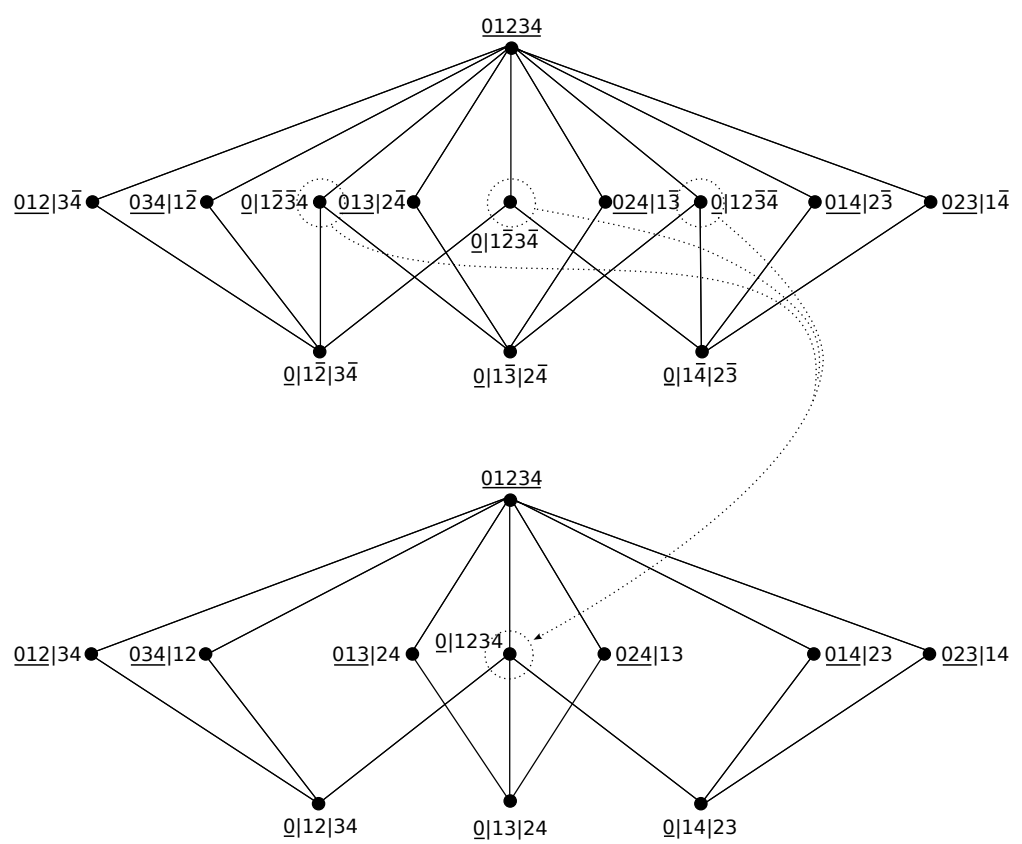

FiguRE 11. The zeroing map $\Pi_{4}^{2} \rightarrow \Pi_{4}^{(2)}$.

Proposition 9.14. The map $Z: \Pi_{n}^{d} \rightarrow \Pi_{n}^{(d)} \subseteq \Pi_{n, 1}$ given by zeroing each block

$$
Z: B_{0} / B_{1} / \ldots / B_{\ell} \mapsto B_{0} / B_{1}^{\text {zero }} / \ldots / B_{\ell}^{\text {zero }}
$$

is a rank-preserving surjective $\mathfrak{S}_{n}$-poset map.

Conjecture 9.15. Let $Z_{*}: \widetilde{H}_{a(d)-1}\left(\Pi_{n}^{d} \backslash\{\hat{1}\}\right) \rightarrow \widetilde{H}_{a(d)-1}\left(\Pi_{n}^{(d)} \backslash\{\hat{1}\}\right)$ be the homomorphism induced by the poset map of Proposition 9.14. Then $Z_{*}$ is surjective. 
Note that by Theorem 9.12 the conjecture is trivially true when $d \mid n$. The conjecture also clearly holds when $r\left(\Pi_{n}^{d}\right)=1$, i.e., when $a(d)=1$. In fact, the entire top homology module is straightforward in this case:

Proposition 9.16. Suppose $a(d)=1$. Let $C_{d}$ be the cyclic group generated by the $d$-cycle $(1,2, \ldots, d)$ and let $\mathfrak{S}_{n-d}=\mathfrak{S}_{\{d+1, d+2, \ldots, n\}}$. Then

$$
\widetilde{H}_{a(d)-1}\left(\Pi_{n}^{d} \backslash\{\hat{1}\}\right) \cong_{\mathfrak{S}_{n}}\left(\operatorname{Ind}_{C_{d} \times \mathfrak{S}_{n-d}}^{\mathfrak{S}_{n}} \mathbb{1}\right)-\mathbb{1} .
$$

Proof. $\mathfrak{S}_{n}$ acts transitively on the elements of $\Pi_{n}^{d} \backslash\{\hat{1}\}$ in this case, and $C_{d} \times \mathfrak{S}_{n-d}$ is the stabilizer of the distinguished element of Proposition 9.3 . Hence the result.

\section{Proof of Theorem 1.3}

Here we characterize when eigenspace arrangements are actually hyperplane arrangements (Lemma 10.3 below). Then as a consequence of the characterization, Theorem 1.3 follows from results of Bessis and Terao.

The motivating case $d=1$ of Theorem 1.3 has a long history, starting with Fadell and Neuwirth [14] showing that $\mathcal{M}\left(\mathfrak{S}_{n}, 1\right)$ is $K(\pi, 1)$. Deligne [11] later proved Brieskorn's conjecture that $\mathcal{M}(W, 1)$ is $K(\pi, 1)$ for all (complexified) real reflection groups. Then Orlik-Solomon [30] showed that $\mathcal{M}(W, 1)$ is $K(\pi, 1)$ for every Shephard group $W$ (see [31, §6.6]) by showing that $\mathcal{M}(W, 1) / W$ is the same as $\mathcal{M}\left(W^{\prime}, 1\right) / W^{\prime}$ for some associated real reflection group $W^{\prime}$ and invoking Deligne's result, while Nakamura [28] established the result for the family $G(m, p, n)$, leaving only six exceptional cases. Bessis 2 resolved the question in the remaining cases while also recovering many of the previous results with his approach. In particular, he obtained a new proof of Deligne's result for real reflection groups.

Theorem 10.1 (Bessis). For any reflection group $W$, the reflection complement $\mathcal{M}(W, 1)$ is $K(\pi, 1)$, i.e., the universal cover of $\mathcal{M}(W, 1)$ is contractible.

The most one could hope for in general is that $\mathcal{M}(W, d)$ is $K(\pi, 1)$ whenever $a(d)$ is $n$ or $n-1$, since any complex arrangement $\mathcal{A}$ whose complement is $K(\pi, 1)$ must contain a hyperplane; see [7, Cor. 3.2] for a much stronger result. Since Proposition 3.7(1) tells us that $\mathcal{A}(W, d)$ is in fact a hyperplane arrangement when $a(d)$ is $n$ or $n-1$, one may also hope it is free in the sense of Terao [43, who established freeness for reflection arrangements $\mathcal{A}(W, 1)$.

Theorem 10.2 (Terao). For any reflection group $W$, the reflection arrangement $\mathcal{A}(W, 1)$ is free.

Recall that a collection of hyperplanes $\mathcal{A}$ in a vector space $V$ is a free arrangement if the $S$-module of derivations $\delta: S \rightarrow S$ that satisfy $\delta\left(\alpha_{H}\right) \in \alpha_{H} S$ for every hyperplane $H=\operatorname{ker}\left(\alpha_{H}\right)$ in $\mathcal{A}$ is free, where $S=S\left(V^{*}\right)$ as in 33 In particular, the null arrangement $\mathcal{N}_{V}$ of $V$, which contains no subspaces at all, is a free arrangement. Less obvious is that any finite collection of lines through the origin in $\mathbb{C}^{2}$ is free, which follows from Saito's criterion. (It is also straightforward [31, Examples 4.20 and 5.4] to see that they are $K(\pi, 1)$, by which we mean that their complements have the property.) Lastly, recall that the product

$$
\mathcal{A}_{1} \times \mathcal{A}_{2}:=\left\{H_{1} \oplus V_{2}: H_{1} \in \mathcal{A}_{1}\right\} \cup\left\{V_{1} \oplus H_{2}: H_{2} \in \mathcal{A}_{2}\right\}
$$

of two arrangements is free if and only if both $\mathcal{A}_{1}$ and $\mathcal{A}_{2}$ are free; see [31. 
We first characterize when $\mathcal{A}(W, d)$ is a hyperplane arrangement.

Lemma 10.3. Let $W$ be a complex reflection group and let $d>0$.

(i) If $a(d)=n$, then $\mathcal{A}(W, d)=\mathcal{A}(W, 1)$ by Corollary 3.8 .

(ii) If $a(d)=n-1$ and $W$ is irreducible, then one of the following holds:

a. $W$ has rank 2 , so that $\mathcal{A}(W, d)$ is a set of lines through the origin in $\mathbb{C}^{2}$.

b. $W=G_{25}, d=2$ (or 6$)$, and $\mathcal{A}(W, d)=\mathcal{A}\left(W^{\prime}, 1\right)$ for $W^{\prime}=G(3,3,3)$.

c. $W=W\left(A_{3}\right), d=2$, and $\mathcal{A}(W, d)$ is defined by $z_{1} z_{2} z_{3}$.

d. $W=G(m, p, n), d \mid m$ and $d \nmid \frac{n m}{p}$, and $\mathcal{A}(W, d)$ is defined by $z_{1} z_{2} \cdots z_{n}$.

Proof. (ii) is clear. Consulting the classification [34, one finds that the pairs $(W, d)$ listed in (iii) are the only cases such that $a(d)=n-1$. Case (iia) is clear. For (iic) and (iid), set $f_{i}:=e_{i}\left(z_{1}^{m}, z_{2}^{m}, \ldots, z_{n}^{m}\right)$ for $1 \leq i \leq n-1$ and set $f_{n}:=e_{n}\left(z_{1}^{m / p}, z_{2}^{m / p}, \ldots, z_{n}^{m / p}\right)$, where here $e_{i}$ denotes the $i$ th elementary symmetric polynomial in $n$ variables. These form a set of basic invariants for $G(m, p, n)$, the degrees of which are $m, 2 m, \ldots,(n-1) m, n m / p$, and only the last invariant polynomial $f_{n}=\left(z_{1} z_{2} \cdots z_{n}\right)^{m / p}$ has degree $d_{n}=n m / p$ not divisible by $d$. Applying Proposition 3.5 thus gives (iic) and (iid). Similarly, for $G_{25}$ we may choose [26, Eq. 9] coordinates and basic invariants $f_{1}, f_{2}, f_{3}$ (of degrees 6,9,12) such that $f_{2}$ is the polynomial $\left(z_{1}^{3}-z_{2}^{3}\right)\left(z_{1}^{3}-z_{3}^{3}\right)\left(z_{2}^{3}-z_{3}^{3}\right)$ that (by (5) ) defines $\mathcal{A}\left(W^{\prime}, 1\right)$ and then apply Proposition 3.5 .

Now Theorem 1.3 follows from the results of Bessis and Terao.

Proof of Theorem 1.3. As noted above, if $\mathcal{A}(W, d)$ is $K(\pi, 1)$ (resp. free), then either $a(d)=n$ or $a(d)=n-1$. When $a(d)=n$, the converse follows from Theorem 10.1 (resp. Theorem 10.2), so it remains to see that the converse also holds when $a(d)=n-1$. In this case, decompose $W$ and $V:=\mathbb{C}^{n}$ as in Proposition 3.1 so that $W \cong W_{1} \times \cdots \times W_{k}$. Then $\mathcal{A}(W, d)=\mathcal{A}\left(W_{i}, d\right) \times \mathcal{N}_{V_{i} \perp}$ for some index $i$, and thus $\mathcal{A}(W, d)$ is $K(\pi, 1)$ (resp. free) if and only if $\mathcal{A}\left(W_{i}, d\right)$ is $K(\pi, 1)$ (resp. free). Now employ Lemma 10.3(ii).

Remark 10.4. V. Reiner pointed out that one may alternatively consider the question of real $K(\pi, 1)$ complements. That is, for which (uncomplexified) real reflection groups $W \subset \mathrm{GL}\left(\mathbb{R}^{n}\right)$ is the real complement

$$
\mathcal{M}_{\mathbb{R}}(W, 2) \stackrel{\text { def }}{=} \mathbb{R}^{n} \backslash \bigcup_{g \in W} V(g,-1)
$$

a $K(\pi, 1)$ space? Because such a complement is $K(\pi, 1)$ only if the arrangement has codimension 2 , one reduces the question to Weyl groups $W\left(A_{4}\right), W\left(A_{5}\right)$, and $W\left(E_{6}\right)$ via the classification. Though we have not explored these cases, we note that a similar question was answered in [10], where Davis, Januszkiewicz, and Scott established a conjecture of Khovanov [20] asserting that the real complement of any $W$-invariant codimension-2 subarrangement of $\mathscr{L}_{W}$ is $K(\pi, 1)$ when $W$ is a real reflection group. Björner first suggested looking at real $K(\pi, 1)$ complements, and Khovanov was motivated by (and answered positively) Björner's question [3, §13.7] asking whether the complement of the 3-equal arrangement

$$
\mathcal{A}=\left\{\mathbf{x} \in \mathbb{R}^{n}: x_{i}=x_{j}=x_{k} \quad \text { for some triple } i<j<k\right\}
$$

is a $K(\pi, 1)$ space. 


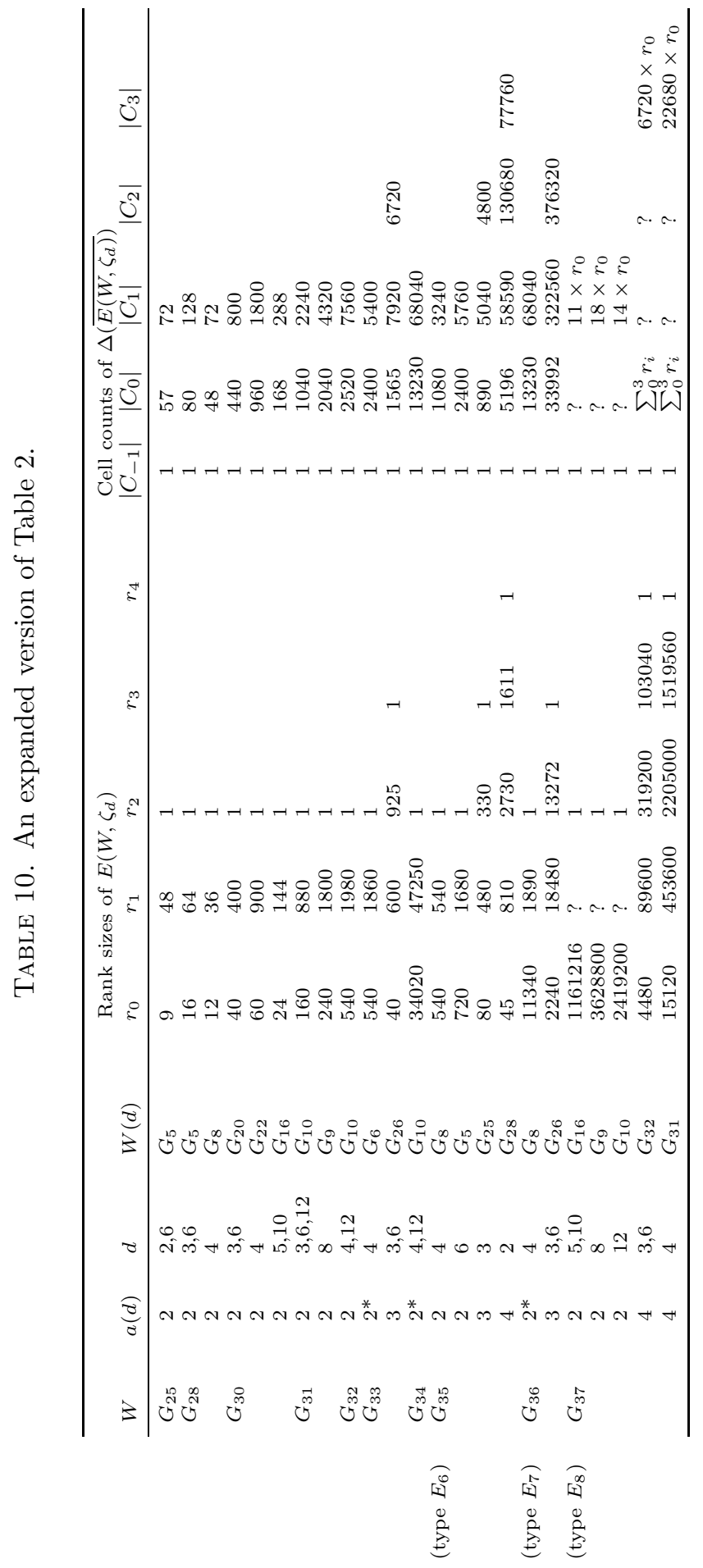




\section{ACKNOWLEDGEMENTS}

This work was partly supported by NSF grant DMS-1001933. The author thanks Victor Reiner for suggestions on the exposition of the material in this paper and for bringing the $K(\pi, 1)$ and freeness questions to his attention. The author is also grateful to the referee for valuable suggestions, and to Anders Björner and Volkmar Welker for their helpful comments and enthusiasm. After this paper was first circulated, Justin Koonin kindly informed us that he was preparing results which are similar to some of ours.

\section{REFERENCES}

[1] Nantel Bergeron, A hyperoctahedral analogue of the free Lie algebra, J. Combin. Theory Ser. A 58 (1991), no. 2, 256-278, DOI 10.1016/0097-3165(91)90061-K. MR.1129117 (93g:20015)

[2] David Bessis, Finite complex reflection arrangements are $K(\pi, 1)$, Ann. of Math. (2) 181 (2015), no. 3, 809-904. MR.3296817

[3] Anders Björner, Subspace arrangements, First European Congress of Mathematics, Vol. I (Paris, 1992), Progr. Math., vol. 119, Birkhäuser, Basel, 1994, pp. 321-370. MR1341828 (96h:52012)

[4] A. Björner, Topological methods, Handbook of combinatorics, Vol. 1, 2, Elsevier, Amsterdam, 1995, pp. 1819-1872. MR1373690 (96m:52012)

[5] Anders Björner and Michelle Wachs, On lexicographically shellable posets, Trans. Amer. Math. Soc. 277 (1983), no. 1, 323-341, DOI 10.2307/1999359. MR690055 (84f:06004)

[6] Anders Björner and Michelle L. Wachs, Shellable nonpure complexes and posets. II, Trans. Amer. Math. Soc. 349 (1997), no. 10, 3945-3975, DOI 10.1090/S0002-9947-97-01838-2. MR:1401765 (98b:06008)

[7] Anders Björner and Volkmar Welker, The homology of " $k$-equal" manifolds and related partition lattices, Adv. Math. 110 (1995), no. 2, 277-313, DOI 10.1006/aima.1995.1012. MR:1317619 (95m:52029)

[8] Michel Broué, Reflection groups, braid groups, Hecke algebras, finite reductive groups, Current developments in mathematics, 2000, Int. Press, Somerville, MA, 2001, pp. 1-107. MR.1882533 (2003e:20044)

[9] A. R. Calderbank, P. Hanlon, and R. W. Robinson, Partitions into even and odd block size and some unusual characters of the symmetric groups, Proc. London Math. Soc. (3) 53 (1986), no. 2, 288-320, DOI 10.1112/plms/s3-53.2.288. MR850222 (87m:20042)

[10] M. Davis, T. Januszkiewicz, and R. Scott, Nonpositive curvature of blow-ups, Selecta Math. (N.S.) 4 (1998), no. 4, 491-547, DOI 10.1007/s000290050039. MR1668119 (2001f:53078)

[11] Pierre Deligne, Les immeubles des groupes de tresses généralisés (French), Invent. Math. 17 (1972), 273-302. MR0422673 (54 \#10659)

[12] T. A. Dowling, A class of geometric lattices based on finite groups, J. Combinatorial Theory Ser. B 14 (1973), 61-86. MR0307951 (46 \#7066)

[13] Richard Ehrenborg and JiYoon Jung, The topology of restricted partition posets (English, with English and French summaries), 23rd International Conference on Formal Power Series and Algebraic Combinatorics (FPSAC 2011), Discrete Math. Theor. Comput. Sci. Proc., AO, Assoc. Discrete Math. Theor. Comput. Sci., Nancy, 2011, pp. 281-292. MR2820717

[14] Edward Fadell and Lee Neuwirth, Configuration spaces, Math. Scand. 10 (1962), 111-118. MR0141126 (25 \#4537)

[15] Mark Goresky and Robert MacPherson, Stratified Morse theory, Ergebnisse der Mathematik und ihrer Grenzgebiete (3) [Results in Mathematics and Related Areas (3)], vol. 14, SpringerVerlag, Berlin, 1988. MR932724 (90d:57039)

[16] Eric Gottlieb and Michelle L. Wachs, Cohomology of Dowling lattices and Lie (super)algebras, Adv. in Appl. Math. 24 (2000), no. 4, 301-336, DOI 10.1006/aama.1999.0670. MR.1761776 (2001i:05161)

[17] Phil Hanlon, The fixed-point partition lattices, Pacific J. Math. 96 (1981), no. 2, 319-341. MR637975 (83d:06010) 
[18] Phil Hanlon, The characters of the wreath product group acting on the homology groups of the Dowling lattices, J. Algebra 91 (1984), no. 2, 430-463, DOI 10.1016/0021-8693(84)90113-3. MR769584 (86j:05046)

[19] André Joyal, Foncteurs analytiques et espèces de structures (French), Combinatoire énumérative (Montreal, Que., 1985/Quebec, Que., 1985), Lecture Notes in Math., vol. 1234, Springer, Berlin, 1986, pp. 126-159, DOI 10.1007/BFb0072514. MR927763 (89b:05014)

[20] Mikhail Khovanov, Real $K(\pi, 1)$ arrangements from finite root systems, Math. Res. Lett. 3 (1996), no. 2, 261-274, DOI 10.4310/MRL.1996.v3.n2.a11. MR1386845(97d:52023)

[21] A. A. Klyachko, Lie elements in the tensor algebra, Siberian Math. J. 15 (1974), 914-920.

[22] G. I. Lehrer and Louis Solomon, On the action of the symmetric group on the cohomology of the complement of its reflecting hyperplanes, J. Algebra 104 (1986), no. 2, 410-424, DOI 10.1016/0021-8693(86)90225-5. MR866785(88a:32017)

[23] G. I. Lehrer and T. A. Springer, Intersection multiplicities and reflection subquotients of unitary reflection groups. I, Geometric group theory down under (Canberra, 1996), de Gruyter, Berlin, 1999, pp. 181-193. MR.1714845 (2000i:20064)

[24] G. I. Lehrer and T. A. Springer, Reflection subquotients of unitary reflection groups, Dedicated to H. S. M. Coxeter on the occasion of his 90th birthday. Canad. J. Math. 51 (1999), no. 6, 1175-1193, DOI 10.4153/CJM-1999-052-4. MR1756877 (2001f:20082)

[25] Gustav I. Lehrer and Donald E. Taylor, Unitary reflection groups, Australian Mathematical Society Lecture Series, vol. 20, Cambridge University Press, Cambridge, 2009. MR 2542964 (2010j:20056)

[26] Heinrich Maschke, Aufstellung des vollen Formensystems einer quaternären Gruppe von 51840 linearen Substitutionen (German), Math. Ann. 33 (1889), no. 3, 317-344, DOI 10.1007/BF01443964. MR1510546

[27] James R. Munkres, Topological results in combinatorics, Michigan Math. J. 31 (1984), no. 1, 113-128, DOI 10.1307/mmj/1029002969. MR736476 (85k:13022)

[28] Tokushi Nakamura, A note on the $K(\pi, 1)$ property of the orbit space of the unitary reflection group $G(m, l, n)$, Sci. Papers College Arts Sci. Univ. Tokyo 33 (1983), no. 1, 1-6. MR.714667 (85e:32015)

[29] Peter Orlik and Louis Solomon, Unitary reflection groups and cohomology, Invent. Math. 59 (1980), no. 1, 77-94, DOI 10.1007/BF01390316. MR.575083 (81f:32017)

[30] Peter Orlik and Louis Solomon, Discriminants in the invariant theory of reflection groups, Nagoya Math. J. 109 (1988), 23-45. MR931949 (89i:32025)

[31] Peter Orlik and Hiroaki Terao, Arrangements of hyperplanes, Grundlehren der Mathematischen Wissenschaften [Fundamental Principles of Mathematical Sciences], vol. 300, SpringerVerlag, Berlin, 1992. MR 1217488 (94e:52014)

[32] Daniel Quillen, Homotopy properties of the poset of nontrivial p-subgroups of a group, Adv. in Math. 28 (1978), no. 2, 101-128, DOI 10.1016/0001-8708(78)90058-0. MR 493916 (80k:20049)

[33] Bruce E. Sagan, Shellability of exponential structures, Order 3 (1986), no. 1, 47-54, DOI 10.1007/BF00403409. MR850397 (87j:05020)

[34] G. C. Shephard and J. A. Todd, Finite unitary reflection groups, Canadian J. Math. 6 (1954), 274-304. MR0059914(15,600b)

[35] T. A. Springer, Regular elements of finite reflection groups, Invent. Math. 25 (1974), 159-198. MR0354894 (50 \#7371)

[36] Richard P. Stanley, Enumerative combinatorics. Vol. 2, with a foreword by Gian-Carlo Rota and appendix 1 by Sergey Fomin, Cambridge Studies in Advanced Mathematics, vol. 62, Cambridge University Press, Cambridge, 1999. MR.1676282 (2000k:05026)

[37] Richard P. Stanley, Exponential structures, Stud. Appl. Math. 59 (1978), no. 1, $73-82$. MR0480063 (58 \#262)

[38] Richard P. Stanley, Some aspects of groups acting on finite posets, J. Combin. Theory Ser. A 32 (1982), no. 2, 132-161, DOI 10.1016/0097-3165(82)90017-6. MR654618 (83d:06002)

[39] Robert Steinberg, Differential equations invariant under finite reflection groups, Trans. Amer. Math. Soc. 112 (1964), 392-400. MR0167535 (29 \#4807)

[40] Sheila Sundaram, The homology representations of the symmetric group on CohenMacaulay subposets of the partition lattice, Adv. Math. 104 (1994), no. 2, 225-296, DOI 10.1006/aima.1994.1030. MR.1273390 (96c:05189) 
[41] Sheila Sundaram and Volkmar Welker, Group actions on arrangements of linear subspaces and applications to configuration spaces, Trans. Amer. Math. Soc. 349 (1997), no. 4, 1389 1420, DOI 10.1090/S0002-9947-97-01565-1. MR1340186 (97h:52012)

[42] Garrett Smith Sylvester, Continuous-spin Ising ferromagnets, ProQuest LLC, Ann Arbor, MI. Thesis (Ph.D.)-Massachusetts Institute of Technology, 1976. MR2940687

[43] Hiroaki Terao, Free arrangements of hyperplanes and unitary reflection groups, Proc. Japan Acad. Ser. A Math. Sci. 56 (1980), no. 8, 389-392. MR596011(82e:32018a)

[44] Michelle L. Wachs, A basis for the homology of the d-divisible partition lattice, Adv. Math. 117 (1996), no. 2, 294-318, DOI 10.1006/aima.1996.0014. MR.1371655(97a:05216)

[45] Michelle L. Wachs, Poset topology: tools and applications, Geometric combinatorics, IAS/Park City Math. Ser., vol. 13, Amer. Math. Soc., Providence, RI, 2007, pp. 497-615. MR2383132

[46] James W. Walker, Canonical homeomorphisms of posets, European J. Combin. 9 (1988), no. 2, 97-107. MR939858 (89g:06012)

School of Mathematics, University of Minnesota, Minneapolis, Minnesota 55455

Current address: Department of Mathematics, University of Illinois at Urbana-Champaign, Urbana, Illinois 61801

E-mail address: arm@illinois.edu 